PAPER • OPEN ACCESS

\section{A muon-track reconstruction exploiting stochastic losses for large-scale Cherenkov detectors}

To cite this article: on behalf of the IceCube collaboration et al 2021 JINST 16 P08034

View the article online for updates and enhancements.
You may also like

- LOCALIZATION AND BROADBAND
$\frac{\text { FOLLOW-UP OF THE GRAVITATIONAL- }}{\text { WAVE TRANSIENT GW150914 }}$
B. P. Abbott, R. Abbott, T. D. Abbott et al.
- GW190814: Gravitational Waves from the
$\frac{\text { Coalescence of a } 23 \text { Solar Mass Black }}{\text { Hole with a } 2.6 \text { Solar Mass Compact }}$
$\frac{\text { Object }}{\text { R. Abbott, T. D. Abbott, S. Abraham et al. }}$
- Search for Eccentric Binary Black Hole
$\frac{\text { Mergers with Advanced LIGO and }}{\text { Advanced Virgo during Their First and }}$
Second Observing Runs
B. P. Abbott, R. Abbott, T. D. Abbott et al.




\title{
A muon-track reconstruction exploiting stochastic losses for large-scale Cherenkov detectors
}

\author{
R. Abbasi, ${ }^{16}$ M. Ackermann, ${ }^{57}$ J. Adams, ${ }^{17}$ J.A. Aguilar, ${ }^{11}$ M. Ahlers, ${ }^{21}$ M. Ahrens,${ }^{48}$ \\ C. Alispach, ${ }^{27}$ A.A. Alves Jr., ${ }^{30}$ N.M. Amin, ${ }^{40}$ R. An, ${ }^{13}$ K. Andeen, ${ }^{38}$ T. Anderson, ${ }^{54}$ \\ I. Ansseau, ${ }^{11}$ G. Anton, ${ }^{25}$ C. Argüelles,,${ }^{13}$ S. Axani, ${ }^{14}$ X. Bai, ${ }^{44}$ A. Balagopal V., ${ }^{36}$
} A. Barbano, ${ }^{27}$ S.W. Barwick, ${ }^{29}$ B. Bastian,,${ }^{57}$ V. Basu, ${ }^{36}$ S. Baur, ${ }^{11}$ R. Bay, ${ }^{7}$ J.J. Beatty, $,{ }^{19}, 20$ K.-H. Becker, ${ }^{56}$ J. Becker Tjus, ${ }^{10}$ C. Bellenghi, ${ }^{26}$ S. BenZvi, ${ }^{46}$ D. Berley, ${ }^{18}$ E. Bernardini, ${ }^{57, a}$ D.Z. Besson, ${ }^{31, b}$ G. Binder, ${ }^{7,8}$ D. Bindig, ${ }^{56}$ E. Blaufuss, ${ }^{18}$ S. Blot, ${ }^{57}$ J. Borowka, ${ }^{0}$ S. Böser, ${ }^{37}$ O. Botner, ${ }^{55}$ J. Böttcher, ${ }^{0}$ E. Bourbeau, ${ }^{21}$ J. Bourbeau, ${ }^{36}$ F. Bradascio, ${ }^{57}$ J. Braun, ${ }^{36}$ S. Bron, ${ }^{27}$ J. Brostean-Kaiser, ${ }^{57}$ S. Browne, ${ }^{30}$ A. Burgman, ${ }^{55}$ R.S. Busse,,${ }^{39}$ M.A. Campana, ${ }^{43}$ C. Chen, ${ }^{5}$ D. Chirkin, ${ }^{36}$ K. Choi, ${ }^{50}$ B.A. Clark, ${ }^{23}$ K. Clark, ${ }^{32}$ L. Classen, ${ }^{39}$ A. Coleman, ${ }^{40}$ G.H. Collin, ${ }^{14}$ J.M. Conrad, ${ }^{14}$ P. Coppin, ${ }^{12}$ P. Correa, ${ }^{12}$ D.F. Cowen,${ }^{53,54}$ R. Cross, ${ }^{46}$ P. Dave, ${ }^{5}$ C. De Clercq, ${ }^{12}$ J.J. DeLaunay, ${ }^{54}$ H. Dembinski, ${ }^{40}$ K. Deoskar, ${ }^{48}$ S. De Ridder, ${ }^{28}$ A. Desai, ${ }^{36}$ P. Desiati, ${ }^{36}$ K.D. de Vries, ${ }^{12}$ G. de Wasseige, ${ }^{12}$ M. de With, ${ }^{9}$ T. DeYoung, ${ }^{23}$ S. Dharani, ${ }^{0}$ A. Diaz, ${ }^{14}$ J.C. Díaz-Vélez, ${ }^{36}$ H. Dujmovic,${ }^{30}$ M. Dunkman, ${ }^{54}$ M.A. DuVernois, ${ }^{36}$ E. Dvorak, ${ }^{44}$ T. Ehrhardt, ${ }^{37}$ P. Eller, ${ }^{26}$ R. Engel, ${ }^{30}$ H. Erpenbeck, ${ }^{0}$ J. Evans, ${ }^{18}$ P.A. Evenson, ${ }^{40}$ S. Fahey,,${ }^{36}$ A.R. Fazely, ${ }^{6}$ S. Fiedlschuster, ${ }^{25}$ A.T. Fienberg, ${ }^{54}$ K. Filimonov, ${ }^{7}$ C. Finley, ${ }^{48}$ L. Fischer, ${ }^{57}$ D. Fox, ${ }^{53}$ A. Franckowiak, ${ }^{10,57}$ E. Friedman, ${ }^{18}$ A. Fritz, ${ }^{37}$ P. Fürst,${ }^{0}$ T. K. Gaisser, ${ }^{40}$ J. Gallagher,,${ }^{35}$ E. Ganster, ${ }^{0}$ S. Garrappa, ${ }^{57}$ L. Gerhardt, ${ }^{8}$ A. Ghadimi, ${ }^{52}$ C. Glaser,,${ }^{55}$ T. Glauch, ${ }^{26}$ T. Glüsenkamp, ${ }^{25}$ A. Goldschmidt, ${ }^{8}$ J.G. Gonzalez, ${ }^{40}$ S. Goswami, ${ }^{52}$ D. Grant, ${ }^{23}$ T. Grégoire, ${ }^{54}$ Z. Griffith, ${ }^{36}$ S. Griswold, ${ }^{46}$ M. Gündüz, ${ }^{10}$ C. Günther, ${ }^{0}$ C. Haack, ${ }^{26}$ A. Hallgren, ${ }^{55}$ R. Halliday, ${ }^{23}$ L. Halve, ${ }^{0}$ F. Halzen,,${ }^{36}$ M. Ha Minh, ${ }^{26}$ K. Hanson, ${ }^{36}$ J. Hardin,,${ }^{36}$ A.A. Harnisch, ${ }^{23}$ A. Haungs,${ }^{30}$ S. Hauser, ${ }^{0}$ D. Hebecker, ${ }^{9}$ K. Helbing, ${ }^{56}$ F. Henningsen, ${ }^{26}$ E.C. Hettinger, ${ }^{23}$ S. Hickford, ${ }^{56}$ J. Hignight, ${ }^{24}$ C. Hill, ${ }^{15}$ G.C. Hill, ${ }^{1}$ K.D. Hoffman,,${ }^{18}$ R. Hoffmann, ${ }^{56}$ T. Hoinka, ${ }^{22}$ B. Hokanson-Fasig, ${ }^{36}$ K. Hoshina, ${ }^{36, c}$ F. Huang, ${ }^{54}$ M. Huber, ${ }^{26}$ T. Huber, ${ }^{30}$ K. Hultqvist, ${ }^{48}$ M. Hünnefeld, ${ }^{22}$ R. Hussain,,${ }^{36}$ S. In, ${ }^{50}$ N. lovine,${ }^{11}$ A. Ishihara,,${ }^{15}$ M. Jansson, ${ }^{48}$ G.S. Japaridze, ${ }^{4}$ M. Jeong, ${ }^{50}$ B.J.P. Jones, ${ }^{3}$ R. Joppe, ${ }^{0}$ D. Kang, ${ }^{30}$ W. Kang, ${ }^{50}$ X. Kang, ${ }^{43}$ A. Kappes, ${ }^{39}$ D. Kappesser, ${ }^{37}$ T. Karg, ${ }^{57}$ M. Karl, ${ }^{26}$ A. Karle, ${ }^{36}$ U. Katz, ${ }^{25}$ M. Kauer, ${ }^{36}$ M. Kellermann, ${ }^{0}$ J.L. Kelley, ${ }^{36}$ A. Kheirandish, ${ }^{54}$ K. Kin, ${ }^{15}$ T. Kintscher,,${ }^{57}$

\footnotetext{
${ }^{a}$ Also at Università di Padova, I-35131 Padova, Italy.

${ }^{b}$ Also at National Research Nuclear University, Moscow Engineering Physics Institute (MEPhI), Moscow 115409, Russia.

${ }^{\mathrm{c}}$ Also at Earthquake Research Institute, University of Tokyo, Bunkyo, Tokyo 113-0032, Japan.
} 
J. Kiryluk, ${ }^{49}$ S.R. Klein,,${ }^{7,8}$ R. Koirala, ${ }^{40}$ H. Kolanoski, ${ }^{9}$ L. Köpke, ${ }^{37}$ C. Kopper, ${ }^{23}$ S. Kopper, ${ }^{52}$ D.J. Koskinen, ${ }^{21}$ P. Koundal, ${ }^{30}$ M. Kovacevich, ${ }^{43}$ M. Kowalski, ${ }^{9,57}$ K. Krings, ${ }^{26}$ N. Kurahashi, ${ }^{43}$ A. Kyriacou, ${ }^{1}$ C. Lagunas Gualda, ${ }^{57}$ J.L. Lanfranchi, ${ }^{54}$ M.J. Larson, ${ }^{18}$ F. Lauber, ${ }^{56}$ J.P. Lazar, ${ }^{13,36}$ J.W. Lee, ${ }^{50}$ K. Leonard, ${ }^{36}$ A. Leszczyńska, ${ }^{30}$ Y. Li, ${ }^{54}$ Q.R. Liu, ${ }^{36}$ E. Lohfink, ${ }^{37}$ C.J. Lozano Mariscal, ${ }^{39}$ L. Lu, ${ }^{15}$ F. Lucarelli, ${ }^{27}$ A. Ludwig, ${ }^{23,33}$ W. Luszczak, ${ }^{36}$ Y. Lyu, ${ }^{7,8}$ W.Y. Ma, ${ }^{57}$ J. Madsen, ${ }^{36}$ K.B.M. Mahn, ${ }^{23}$ Y. Makino, ${ }^{36}$ S. Mancina, ${ }^{36}$ I.C. Mariş,,${ }^{11}$

R. Maruyama, ${ }^{41}$ K. Mase, ${ }^{15}$ F. McNally, ${ }^{34}$ K. Meagher, ${ }^{36}$ A. Medina, ${ }^{20}$ M. Meier,,${ }^{15}$

S. Meighen-Berger, ${ }^{26}$ J. Merz, ${ }^{0}$ J. Micallef, ${ }^{23}$ D. Mockler, ${ }^{11}$ T. Montaruli, ${ }^{27}$ R.W. Moore, ${ }^{24}$ R. Morse,${ }^{36}$ M. Moulai, ${ }^{14}$ R. Naab, ${ }^{57}$ R. Nagai, ${ }^{15}$ U. Naumann, ${ }^{56}$ J. Necker, ${ }^{57}$ L.V. Nguyễn, ${ }^{23}$ H. Niederhausen, ${ }^{26}$ M.U. Nisa, ${ }^{23}$ S.C. Nowicki, ${ }^{23}$ D.R. Nygren, ${ }^{8}$ A. Obertacke Pollmann, ${ }^{56}$ M. Oehler, ${ }^{30}$ A. Olivas, ${ }^{18}$ E. O'Sullivan, ${ }^{55}$ H. Pandya, ${ }^{40}$ D.V. Pankova, ${ }^{54}$ N. Park, ${ }^{36}$ G.K. Parker, ${ }^{3}$ E.N. Paudel, ${ }^{40}$ L. Paul, ${ }^{38}$ C. Pérez de los Heros,,${ }^{55}$ S. Philippen, ${ }^{0}$ D. Pieloth, ${ }^{22}$ S. Pieper, ${ }^{56}$ A. Pizzuto, ${ }^{36}$ M. Plum,${ }^{38}$ Y. Popovych, ${ }^{37}$ A. Porcelli, ${ }^{28}$ M. Prado Rodriguez, ${ }^{36}$ P.B. Price, ${ }^{7}$ B. Pries, ${ }^{23}$ G.T. Przybylski, ${ }^{8}$ C. Raab,,${ }^{11}$ A. Raissi, ${ }^{17}$ M. Rameez, ${ }^{21}$ K. Rawlins, ${ }^{2}$ I.C. Rea, ${ }^{26}$ A. Rehman, ${ }^{40}$ R. Reimann, ${ }^{0}$ G. Renzi, ${ }^{11}$ E. Resconi, ${ }^{26}$ S. Reusch, ${ }^{57}$ W. Rhode,,${ }^{22}$ M. Richman, ${ }^{43}$ B. Riedel, ${ }^{36}$ S. Robertson, ${ }^{7,8}$ G. Roellinghoff, ${ }^{50}$ M. Rongen, ${ }^{37}$ C. Rott,,${ }^{4750}$ T. Ruhe, ${ }^{22}$ D. Ryckbosch, ${ }^{28}$ D. Rysewyk Cantu, ${ }^{23}$ I. Safa, ${ }^{13,36}$ J. Saffer, ${ }^{30}$

S.E. Sanchez Herrera, ${ }^{23}$ A. Sandrock, ${ }^{22}$ J. Sandroos, ${ }^{37}$ M. Santander, ${ }^{52}$ S. Sarkar, ${ }^{42}$ S. Sarkar, ${ }^{24}$ K. Satalecka, ${ }^{57}$ M. Scharf, ${ }^{0}$ M. Schaufel, ${ }^{0}$ H. Schieler, ${ }^{30}$ P. Schlunder, ${ }^{22}$ T. Schmidt, ${ }^{18}$ A. Schneider, ${ }^{36}$ J. Schneider, ${ }^{25}$ F.G. Schröder, ${ }^{30,40}$ L. Schumacher, ${ }^{0}$ S. Sclafani, ${ }^{43}$ D. Seckel,${ }^{40}$ S. Seunarine,${ }^{45}$ A. Sharma, ${ }^{55}$ S. Shefali, ${ }^{30}$ M. Silva, ${ }^{36}$ B. Skrzypek, ${ }^{13}$ B. Smithers, ${ }^{3}$ R. Snihur, ${ }^{36}$ J. Soedingrekso, ${ }^{22}$ D. Soldin, ${ }^{40}$ G.M. Spiczak, ${ }^{45}$ C. Spiering, ${ }^{57, b}$ J. Stachurska, ${ }^{57}$ M. Stamatikos, ${ }^{20}$ T. Stanev, ${ }^{40}$ R. Stein, ${ }^{57}$ J. Stettner, ${ }^{0}$ A. Steuer, ${ }^{37}$ T. Stezelberger, ${ }^{8}$ T. Stürwald,${ }^{56}$ T. Stuttard, ${ }^{21}$ G.W. Sullivan, ${ }^{18}$ I. Taboada, ${ }^{5}$ F. Tenholt, ${ }^{10}$ S. Ter-Antonyan, ${ }^{6}$ S. Tilav,${ }^{40}$ F. Tischbein,,${ }^{0}$ K. Tollefson, ${ }^{23}$ L. Tomankova, ${ }^{10}$ C. Tönnis, ${ }^{51}$ S. Toscano, ${ }^{11}$ D. Tosi, ${ }^{36}$ A. Trettin, ${ }^{57}$ M. Tselengidou, ${ }^{25}$ C.F. Tung,,${ }^{5}$ A. Turcati, ${ }^{26}$ R. Turcotte, ${ }^{30}$ C.F. Turley, ${ }^{54}$ J.P. Twagirayezu, ${ }^{23}$ B. Ty, ${ }^{36}$ M.A. Unland Elorrieta, ${ }^{39}$ N. Valtonen-Mattila, ${ }^{55} \mathrm{~J}$. Vandenbroucke, ${ }^{36} \mathrm{D}$. van Eijk, ${ }^{36} \mathrm{~N}$. van Eijndhoven, ${ }^{12}$ D. Vannerom, ${ }^{14}$ J. van Santen, ${ }^{57}$ S. Verpoest, ${ }^{28}$ M. Vraeghe,${ }^{28}$ C. Walck, ${ }^{48}$ A. Wallace, ${ }^{1}$ T.B. Watson, ${ }^{3}$ C. Weaver, ${ }^{23}$ P. Weigel, ${ }^{14}$ A. Weindl,,${ }^{30}$ M.J. Weiss, ${ }^{54}$ J. Weldert, ${ }^{37}$ C. Wendt, ${ }^{36}$ J. Werthebach, ${ }^{22}$ M. Weyrauch, ${ }^{30}$ B.J. Whelan, ${ }^{1}$ N. Whitehorn, ${ }^{23,33}$ C.H. Wiebusch, ${ }^{0}$ D.R. Williams, ${ }^{52}$ M. Wolf, ${ }^{26}$ K. Woschnagg, ${ }^{7}$ G. Wrede, ${ }^{25}$ J. Wulff, ${ }^{10}$ X.W. Xu, ${ }^{6}$ Y. Xu, ${ }^{49}$ J.P. Yanez, ${ }^{24}$ S. Yoshida, ${ }^{15}$ T. Yuan $^{36}$ and Z. Zhang ${ }^{49}$ on behalf of the IceCube collaboration

${ }^{0}$ III. Physikalisches Institut, RWTH Aachen University, D-52056 Aachen, Germany

${ }^{1}$ Department of Physics, University of Adelaide, Adelaide, 5005, Australia

${ }^{2}$ Department of Physics and Astronomy, University of Alaska Anchorage, 3211 Providence Dr., Anchorage, AK 99508, U.S.A.

${ }^{3}$ Department of Physics, University of Texas at Arlington, 502 Yates St., Science Hall Rm 108, Box 19059, Arlington, TX 76019, U.S.A.

${ }^{4}$ CTSPS, Clark-Atlanta University, Atlanta, GA 30314, U.S.A.

${ }^{5}$ School of Physics and Center for Relativistic Astrophysics, Georgia Institute of Technology, Atlanta, GA 30332, U.S.A.

${ }^{6}$ Department of Physics, Southern University, Baton Rouge, LA 70813, U.S.A. 
${ }^{7}$ Department of Physics, University of California, Berkeley, CA 94720, U.S.A.

${ }^{8}$ Lawrence Berkeley National Laboratory, Berkeley, CA 94720, U.S.A.

${ }^{9}$ Institut für Physik, Humboldt-Universität zu Berlin, D-12489 Berlin, Germany

${ }^{10}$ Fakultät für Physik \& Astronomie, Ruhr-Universität Bochum, D-44780 Bochum, Germany

${ }^{11}$ Université Libre de Bruxelles, Science Faculty CP230, B-1050 Brussels, Belgium

${ }^{12}$ Vrije Universiteit Brussel (VUB), Dienst ELEM, B-1050 Brussels, Belgium

${ }^{13}$ Department of Physics and Laboratory for Particle Physics and Cosmology, Harvard University, Cambridge, MA 02138, U.S.A.

${ }^{14}$ Department of Physics, Massachusetts Institute of Technology, Cambridge, MA 02139, U.S.A.

${ }^{15}$ Department of Physics and Institute for Global Prominent Research, Chiba University, Chiba 263-8522, Japan

${ }^{16}$ Department of Physics, Loyola University Chicago, Chicago, IL 60660, U.S.A.

${ }^{17}$ Department of Physics and Astronomy, University of Canterbury, Private Bag 4800, Christchurch, New Zealand

${ }^{18}$ Department of Physics, University of Maryland, College Park, MD 20742, U.S.A.

${ }^{19}$ Department of Astronomy, Ohio State University, Columbus, OH 43210, U.S.A.

${ }^{20}$ Department of Physics and Center for Cosmology and Astro-Particle Physics, Ohio State University, Columbus, OH 43210, U.S.A.

${ }^{21}$ Niels Bohr Institute, University of Copenhagen, DK-2100 Copenhagen, Denmark

${ }^{22}$ Department of Physics, TU Dortmund University, D-44221 Dortmund, Germany

${ }^{23}$ Department of Physics and Astronomy, Michigan State University, East Lansing, MI 48824, U.S.A.

${ }^{24}$ Department of Physics, University of Alberta, Edmonton, Alberta, Canada T6G $2 E 1$

${ }^{25}$ Erlangen Centre for Astroparticle Physics, Friedrich-Alexander-Universität Erlangen-Nürnberg, D-91058 Erlangen, Germany

${ }^{26}$ Physik-department, Technische Universität München, D-85748 Garching, Germany

${ }^{27}$ Département de physique nucléaire et corpusculaire, Université de Genève, CH-1211 Genève, Switzerland

${ }^{28}$ Department of Physics and Astronomy, University of Gent, B-9000 Gent, Belgium

${ }^{29}$ Department of Physics and Astronomy, University of California, Irvine, CA 92697, U.S.A.

${ }^{30}$ Karlsruhe Institute of Technology, Institute for Astroparticle Physics, D-76021 Karlsruhe, Germany

${ }^{31}$ Department of Physics and Astronomy, University of Kansas, Lawrence, KS 66045, U.S.A.

${ }^{32}$ SNOLAB, 1039 Regional Road 24, Creighton Mine 9, Lively, ON, Canada P3Y 1N2

${ }^{33}$ Department of Physics and Astronomy, UCLA, Los Angeles, CA 90095, U.S.A.

${ }^{34}$ Department of Physics, Mercer University, Macon, GA 31207-0001, U.S.A.

${ }^{35}$ Department of Astronomy, University of Wisconsin-Madison, Madison, WI 53706, U.S.A.

${ }^{36}$ Department of Physics and Wisconsin Ice Cube Particle Astrophysics Center, University of Wisconsin-Madison, Madison, WI 53706, U.S.A.

${ }^{37}$ Institute of Physics, University of Mainz, Staudinger Weg 7, D-55099 Mainz, Germany

${ }^{38}$ Department of Physics, Marquette University, Milwaukee, WI, 53201, U.S.A.

${ }^{39}$ Institut für Kernphysik, Westfälische Wilhelms-Universität Münster, D-48149 Münster, Germany

${ }^{40}$ Bartol Research Institute and Department of Physics and Astronomy, University of Delaware, Newark, DE 19716, U.S.A.

${ }^{41}$ Department of Physics, Yale University, New Haven, CT 06520, U.S.A.

${ }^{42}$ Department of Physics, University of Oxford, Parks Road, Oxford OX1 3PU, U.K.

${ }^{43}$ Department of Physics, Drexel University, 3141 Chestnut Street, Philadelphia, PA 19104, U.S.A. 
${ }^{44}$ Physics Department, South Dakota School of Mines and Technology, Rapid City, SD 57701, U.S.A.

${ }^{45}$ Department of Physics, University of Wisconsin, River Falls, WI 54022, U.S.A.

${ }^{46}$ Department of Physics and Astronomy, University of Rochester, Rochester, NY 14627, U.S.A.

${ }^{47}$ Department of Physics and Astronomy, University of Utah, Salt Lake City, UT 84112, U.S.A.

${ }^{48}$ Oskar Klein Centre and Department of Physics, Stockholm University, SE-10691 Stockholm, Sweden

${ }^{49}$ Department of Physics and Astronomy, Stony Brook University, Stony Brook, NY 11794-3800, U.S.A.

${ }^{50}$ Department of Physics, Sungkyunkwan University, Suwon 16419, Korea

${ }^{51}$ Institute of Basic Science, Sungkyunkwan University, Suwon 16419, Korea

${ }^{52}$ Department of Physics and Astronomy, University of Alabama, Tuscaloosa, AL 35487, U.S.A.

${ }^{53}$ Department of Astronomy and Astrophysics, Pennsylvania State University, University Park, PA 16802, U.S.A.

${ }^{54}$ Department of Physics, Pennsylvania State University, University Park, PA 16802, U.S.A.

${ }^{55}$ Department of Physics and Astronomy, Uppsala University, Box 516, S-75120 Uppsala, Sweden

${ }^{56}$ Department of Physics, University of Wuppertal, D-42119 Wuppertal, Germany

${ }^{57}$ DESY, D-15738 Zeuthen, Germany

E-mail: analysis@icecube.wisc.edu

AbstRact: IceCube is a cubic-kilometer Cherenkov telescope operating at the South Pole. The main goal of IceCube is the detection of astrophysical neutrinos and the identification of their sources. High-energy muon neutrinos are observed via the secondary muons produced in charge current interactions with nuclei in the ice. Currently, the best performing muon track directional reconstruction is based on a maximum likelihood method using the arrival time distribution of Cherenkov photons registered by the experiment's photomultipliers. A known systematic shortcoming of the prevailing method is to assume a continuous energy loss along the muon track. However at energies $>1 \mathrm{TeV}$ the light yield from muons is dominated by stochastic showers. This paper discusses a generalized ansatz where the expected arrival time distribution is parametrized by a stochastic muon energy loss pattern. This more realistic parametrization of the loss profile leads to an improvement of the muon angular resolution of up to $20 \%$ for through-going tracks and up to a factor 2 for starting tracks over existing algorithms. Additionally, the procedure to estimate the directional reconstruction uncertainty has been improved to be more robust against numerical errors.

KeYwords: Cherenkov detectors; Neutrino detectors; Data analysis

ArXIV EPrint: 2103.16931 


\section{Contents}

1 Introduction 1

2 Previous algorithms 2

2.1 Angular reconstruction 2

$\begin{array}{ll}2.2 & \text { Uncertainty estimation }\end{array}$

3 New algorithm: SegmentedSplineReco $\quad 7$

3.1 Angular reconstruction $\quad 8$

$\begin{array}{ll}3.2 & \text { Uncertainty estimation } \\ \end{array}$

4 Performance comparisons $\quad 11$

4.1 Angular resolution 12

$\begin{array}{ll}4.2 & \text { Uncertainty estimation } \\ & 14\end{array}$

$\begin{array}{lll}4.3 & \text { Runtime } & 15\end{array}$

5 Discussion and outlook 16

A Parametrizations of track orientations 17

$\begin{array}{lll}\text { A.1 Tangent plane parametrization } & 17\end{array}$

$\begin{array}{ll}\text { A.2 Rotation to }(1,0,0) & 17\end{array}$

B Technical details of the uncertainty estimation $\quad 18$

\section{Introduction}

The IceCube Neutrino Observatory is a cubic-kilometre neutrino telescope located at the geographic South Pole [1,2]. It consists of 5160 digital optical modules (DOMs), each containing a 10-inch photomultiplier tube (PMT). The PMTs detect Cherenkov photons emitted from charged secondary particles, i.e. electrons and muons, created in neutrino interactions in the surrounding ice. The ice in which IceCube is deployed is of glacial origin and exceptionally pure. However, it contains impurities such as dust and volcanic ash, most prominently in a layer between $\sim 2000 \mathrm{~m}$ and $\sim 2100 \mathrm{~m}$ depth [3-5]. Further irregularities in the ice include bubble columns in the refrozen ice around the strings of DOMs, a tilt of the ice sheet, and an anisotropic attenuation aligned with the local flow of the ice $[6,7]$. A series of light-emitting diodes (LEDs) are used to illuminate the PMTs and parametrize the ice properties [5].

In 2013, the IceCube collaboration detected the first astrophysical neutrinos in the TeV-PeV range [8]. Since then, further studies have been initiated to understand the origin of these neutrinos, e.g. [9-11]. Many of these are point-source studies searching for correlations between an excess in neutrino events and known astrophysical source locations [12]. The coincidence in 2017 of 
a high energy neutrino event and the flaring blazar TXS 0506+056 [13] reinforced the idea of a fraction of blazars being the sources of high-energy neutrinos. In point-source analyses, a precise reconstruction of the direction of the neutrino is a central aspect that contributes most to the detection sensitivity. Apart from time integrated searches that collect large statistics samples, single real-time neutrino alerts that are sent out to the astronomical community also require a precise directional reconstruction [14].

An important detection channel for point source identifications are muons which originate from $v_{\mu}$ charged current (CC) interactions, which appear as track signatures in the IceCube detector. Above a few $\mathrm{TeV}$, these muons are nearly co-aligned with the direction of the parent neutrino due to relativistic kinematics (see section 2 for a more quantitative discussion). At these energies, a confidence interval on the muon arrival direction approximately translates into a confidence interval on the parent neutrino direction.

Muons at energies above $\sim 1 \mathrm{TeV}$ loose their energy mostly stochastically by bremsstrahlung, pair production, and nuclear interactions [15]. Existing muon reconstructions in IceCube directly model only the minimum-ionizing continuous energy loss, while either neglecting the stochastic part or modeling it in an effective manner. This paper describes a new likelihood reconstruction that incorporates these stochastic energy losses into the likelihood hypothesis directly and thereby improves the accuracy and precision of the reconstructed arrival direction. Section 2 summarizes the previous likelihood approaches and lists their limitations. Section 3 introduces the new likelihood approach and discusses how some shortcomings of the previous algorithms are solved. The discussion here concentrates around both the angular reconstruction and uncertainty estimation. Section 4 and section 5 show comparisons of the various methods and conclude this manuscript with a final discussion.

\section{Previous algorithms}

This section illustrates a typical reconstruction process and in this context describes various existing reconstructions that are useful to understand the benefits of the new approach. Some of the details in this section have been already partially covered in refs. [16] and [17].

\subsection{Angular reconstruction}

IceCube collects Cherenkov photons from charged particles with a few nanosecond time resolution at the various DOM locations [2]. This time resolution is required because a Cherenkov light front from a muon passing by a DOM yields a photon arrival time probability distribution function (PDF) that can have temporal structures of this magnitude [18]. This time resolution can best be exploited in an unbinned likelihood approach. The prevailing angular reconstructions typically assume an infinite muon track length and neglect stochastic losses. Such reconstructions employ two broad classes of unbinned likelihood approaches to model the arrival time of photons:

$$
L(\Theta)=\prod_{j=1}^{N_{\mathrm{DOM}}} \prod_{i=1}^{N_{\mathrm{hit}}}\left[p_{j}\left(t_{j, i}\right)\right]^{q_{j, i}}
$$




$$
\begin{aligned}
L_{1 \mathrm{st}}(\Theta) & =\prod_{j=1}^{N_{\mathrm{DOM}}} p_{j, 1 \mathrm{st}}\left(t_{j, 1}\right) \\
& \propto \prod_{j=1}^{N_{\mathrm{DOM}}}\left[p_{j}\left(t_{j, 1}\right)\right]^{q_{j, 1}} \cdot\left(1-P_{j}\left(t_{j, 1}\right)\right)^{Q_{j}-q_{j, 1}}
\end{aligned}
$$

where $N_{\text {DOM }}$ and $N_{\text {hit }}$ are the total number of DOMs and hits, respectively. The $i$-th observed photon in DOM $j$ has a hit time $t_{j, i}$ and a charge $q_{j, i}$. The first likelihood (equation (2.1)) is the standard unbinned likelihood and uses the photon arrival PDF $p(t)$ for each observed hit. Since multiple photons can arrive at the DOM simultaneously, the correct application weights each hit by the observed charge $q_{i}$. In practice, this is often neglected since the effect it has is subdominant compared to the unphysical assumption of the minimally ionizing track hypothesis. The second likelihood (equation (2.2)) uses the photon arrival PDF of only the first photon, $p_{j, 1 \text { st }}\left(t_{1}\right)$, which technically corresponds to the first-order-statistic PDF. ${ }^{1}$ This PDF can be calculated exactly given the PDF $p(t)$ and the cumulative distribution function (CDF) $P(t)$ [16]. The total charge observed in DOM $j$ is denoted by $Q_{j}=\sum_{i} q_{j, i}$. The motivation for the first-order statistic PDF is twofold. While the standard PDF (equation 2.1) is able to model the photon arrival distribution, it can be biased when systematic uncertainties are present. One systematic effect comes from the assumption of a minimally ionizing muon, while muons at energies beyond $\mathrm{TeV}$ energies undergo stochastic energy losses. Additionally, the PDF typically makes simplifying modelling assumptions about the ice properties, for example homogeneity. Both effects are partly mitigated by using $L_{1 \mathrm{st}}$. It evaluates only the first observed photon in each DOM which is less likely to have undergone significant scattering and hence the likelihood is less affected by the exact ice properties. Only using the first photon leads to an overall lower number of counts, and therefore also effectively reduces the bias from the wrong minimal-ionizing track assumption.

The optimization is a 6-dimensional problem for the track positional parameters $x, y, z, t$ and orientation via two angles, e.g. zenith $\theta$ and azimuth $\phi$. We summarize those parameters as $\Theta=(x, y, z, t, \theta, \phi)$. In practice, the angles are sometimes re-parametrized as two parameters lying in the sphere tangent plane that is perpendicular to the initial guess track (seed) direction in order to avoid numerical issues near the poles (see appendix A).

The following sections describe a processing pipeline in which several reconstructions with increasing complexity are applied to the same event (see figure 1). Each reconstruction outcome is used as a seeding strategy to the next one, starting with the fastest and simplest reconstruction and becoming gradually more time consuming and more precise. Note that in practice different processing pipelines are used based on the needs of a given event selection. However, the pipeline illustrated in figure 1 is representative in that it shares the property of starting simple and fast and becoming gradually more time consuming and precise. ${ }^{2}$ Furthermore, it illustrates the thought process that went into the development of the new reconstruction.

Analytic Gaussian PDF (Least-Square) with plane-wave assumption. The "first-guess" fit is typically a least-square fit which does not assume a minimal-ionizing muon, but a plane-wave

\footnotetext{
${ }^{1}$ In a previous publication [16] the likelihood is called "MPE" likelihood instead of $L_{1 \mathrm{st}}$.

${ }^{2}$ There is an exception in the time consumption of $L_{1 s t}$, which only requires the evaluation of the first photon, and is therefore actually faster, while also giving a more precise result.
} 
Previous Algorithms

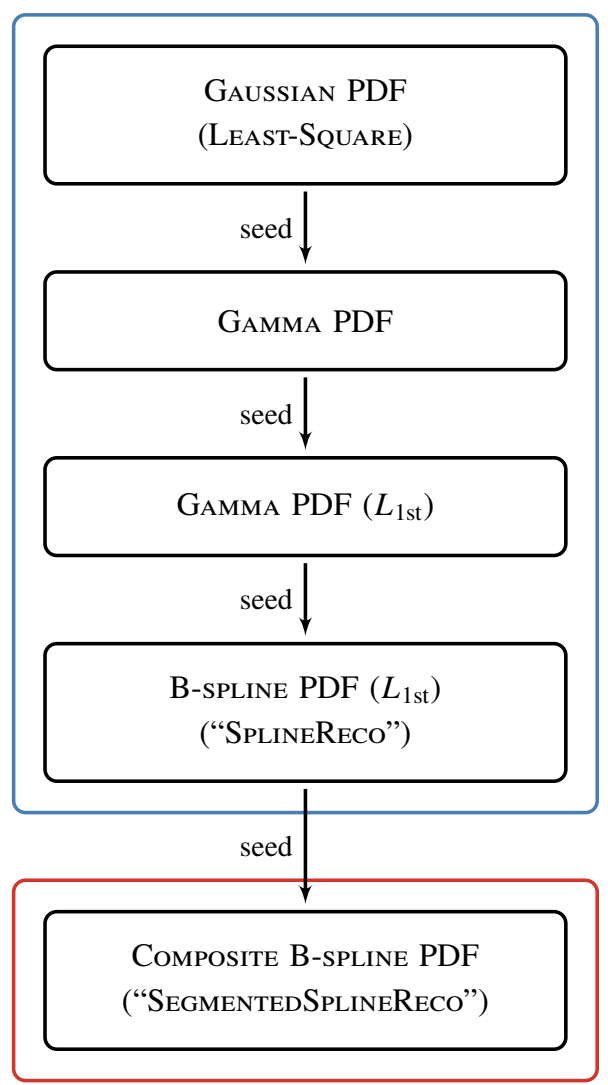

New Algorithm

Figure 1. An example track reconstruction seeding chain depicting the underlying assumed PDF in the respective likelihood reconstruction. The PDF approximation quality and CPU time requirements broadly increase with more sophisticated reconstructions later in the chain.

moving through the detector with a constant velocity. ${ }^{3}$ Assuming a standard normal arrival time PDF of this plane wave one can analytically calculate a mean position and velocity vector of the least-square problem [19]. To partially mitigate this rather simplified approximation a more robust regression with respect to outliers is typically used, for example by the inclusion of a Huber loss term [20]. The robust algorithm is shown in figure 2.

Analytic Gamma PDF. A more physically motivated description of the photon arrival PDF comes from assuming a minimally ionizing muon track instead of a plane wave. The photon arrival PDF $p(t)$ can in this case be approximated with a gamma distribution, as discussed in more detail in $[16],{ }^{4}$ as

$$
p(t)=\frac{\beta^{\alpha} \cdot\left(t-t_{0}\right)^{\alpha-1} \cdot e^{-\beta \cdot\left(t-t_{0}\right)}}{\Gamma(\alpha)}
$$

\footnotetext{
${ }^{3}$ This algorithm has been previously been referred to as "line-fit"[16].

${ }^{4}$ In a previous publication [16] this approximation of the PDF with a gamma distribution has been called "Pandel" approximation.
} 
with $\alpha=\frac{d}{\lambda\left(d, \lambda_{s}, \boldsymbol{\theta}\right)}, \beta=\frac{1}{\tau\left(d, \lambda_{s}, \boldsymbol{\theta}\right)}+\frac{c_{m}}{\lambda_{a}}$ and $t_{0}$ being the first time of photon arrival for unscattered photons from the source. The gamma distribution was originally obtained as the solution to the time arrival distribution of a point-like light emission source [21] at distance $d$ in a transparent medium with scattering length $\lambda_{s}$, absorption length $\lambda_{a}$, speed of light in the medium $c_{m}$, and other nuisance parameters $\boldsymbol{\theta}$ which are determined by a Monte Carlo fit. Later it was found [16, 22] to be also a good approximation to the time-arrival distribution of Cherenkov light from an infinite muon, when the distance to the source $d$ is replaced by an effective distance $d=d_{\text {eff }}\left(d_{\text {perp }}, \eta, \omega\right)$ which is a function of the perpendicular distance between muon track and detection module $d_{\text {perp }}$, the angle between the Cherenkov cone of the muon and the axis that describes the orientation of the detection module $\eta$, and additional nuisance parameters $\omega$ that were also determined by a Monte Carlo fit. A generalization with a convolution with a Gaussian distribution that solves numerical issues when the emission point is close to the module is typically used [23]. By varying the track parameters like position and orientation of the track, the PDF parameters $t_{0}$ and $d_{\text {eff }}$ change in turn, and one can in this way optimize the likelihood function with respect to the original track parameters.

The gamma distribution-based parametrization is more accurate not only because of its more realistic assumptions, but also because it allows to calculate analytically the first-order-statistic PDF and thereby $L_{1 \text { st }}$ (see equation (2.2)). Empirically, the gamma PDF with likelihood $L_{1 \text { st }}$ gives better results in terms of mean angular difference between reconstructed and true muon direction than the Gaussian first guess (least-square fit) or the standard likelihood $L$ using the gamma distribution, as shown in figure 2. As described earlier, this improvement of $L_{1 \mathrm{st}}$ can be understood as partly mitigating the inaccurate modeling assumption of an infinite muon by only looking at the first photon. Also shown is the mean angle between the muon and parent neutrino direction. The mean angular error from the displayed muon reconstructions is well above this intrinsic kinematic deflection, especially above $10-100 \mathrm{TeV}$, which means the kinematic deflection can be neglected for most of the energy range.

B-spline PDF modelling. The previous description using an effective gamma distribution as a PDF is motivated by physical considerations, but is ultimately used because of the empirical observation that it gives a more precise muon reconstruction than the least-square fit. However, since the gamma distribution assumes the optical properties of ice are homogeneous it does a poor job of reproducing the exact photon arrival distribution. Replacing this function with BSplines allows for a more accurate representation of the ice properties. Further improvement can be made by incorporating a more realistic ice model, which involves two steps. First, the ice's optical properties are inferred from fitting an ice model to LED flasher data [5]. Typically, the ice is approximated by horizontally homogeneous layers of equal scattering and absorption lengths each having a thickness of 10-20 m. Second, infinite minimal-ionizing muon tracks are simulated with a given ice model in many different positions and orientations, and the resulting photons are recorded in high-dimensional histograms [24]. These histograms are normalized and fitted with multi-dimensional interpolating B-splines [25] to represent the photon arrival PDF in dependence of the track position and orientation as described in detail in [25]. Given $T$ knot positions, the photon arrival PDF is given by

$$
p(t)=\sum_{i=1}^{T-k-1} w_{i} \cdot B_{i, k}\left(t-t_{0} ; \boldsymbol{\Theta}, \lambda\right)
$$




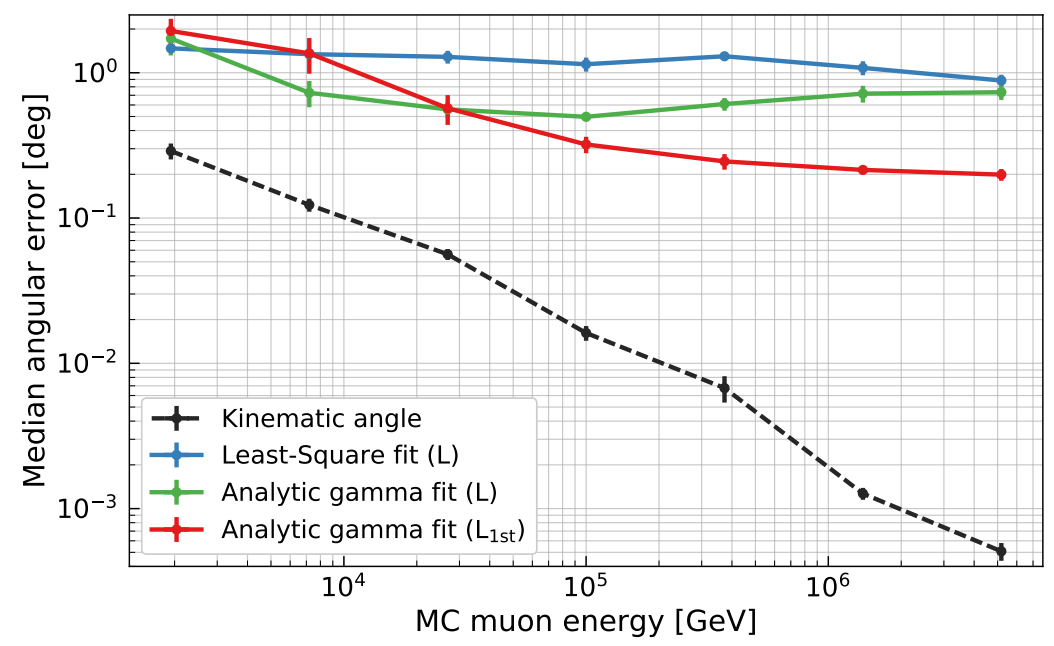

Figure 2. Median angular error for muons passing the whole detector (through-going muons) as a function of the simulated muon energy at the interaction vertex for a least-square fit and two different analytic Gamma fits. The dashed black curve shows the median of the kinematic angle between the neutrino and the muon direction. Each reconstruction is seeded with the previous algorithm, following the chain of figure 1 . The statistical error on the median is calculated using bootstrapping.

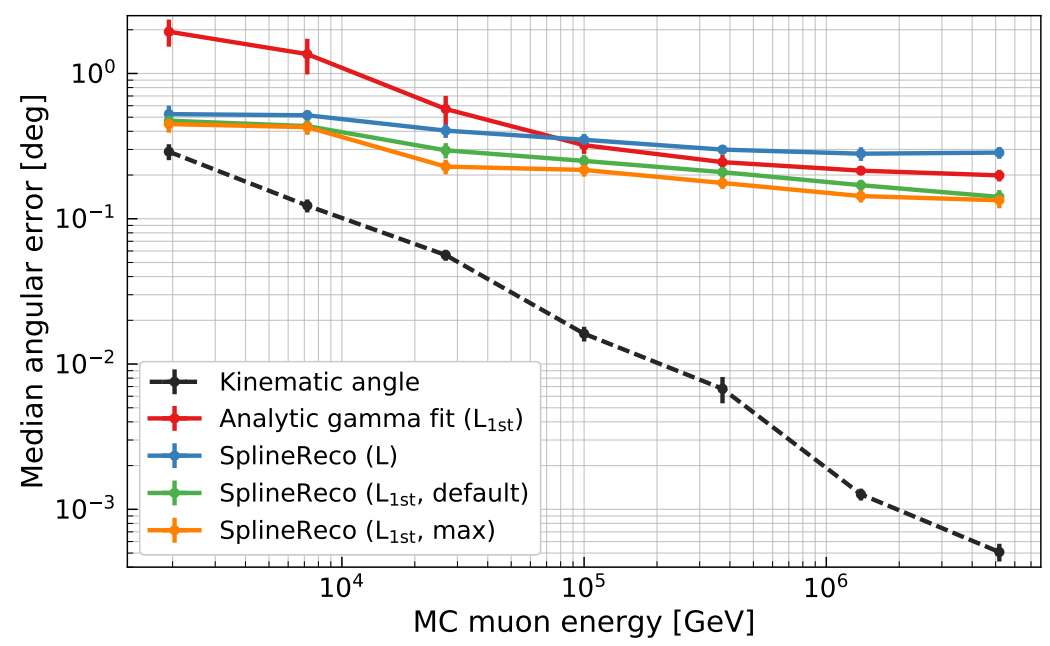

Figure 3. Reconstruction of muons passing the whole detector (through-going muons) using the analytic Gamma fit with the $\mathrm{pdf}_{1 \mathrm{st}}$ PDFs and the SplineReco algorithms. The SplineReco reconstruction is shown using the standard likelihood $L, L_{1 \text { st }}$ with default settings, and $L_{1 s t}$ with max settings. The dashed black curve shows the median of the kinematic angle between the neutrino and the muon direction. Each reconstruction is seeded with the previous best performing algorithm, following the chain of figure 1 . The statistical error on the median is calculated using bootstrapping. The MC muon energy is calculated at the neutrino interaction point.

where $B_{i, k}$ is the $i$-th area-normalized B-spline of order $k, \boldsymbol{\Theta}$ denotes track parameters like the track position and orientation, and $\lambda$ denotes all the ice model parameters. The weights $w_{i}$ sum to unity since each B-spline is by itself normalized in this representation. The parameter $t_{0}=t_{0}(\boldsymbol{\Theta})$ is again determined to be the earliest time a Cherenkov photon can reach the detection module 
without scattering from the track emission point, and thereby also depends on the given track parameters $\boldsymbol{\Theta}$. While PDFs based on B-splines are more flexible and give a better representation of ice properties, the track hypothesis still assumes a minimally-ionizing particle which neglects any stochastic losses of the muon. Several methods have been implemented to mitigate the effects of this unphysical assumption and improve the reconstruction, besides the use of the first-order statistic PDF (usage of $L_{1 \text { st }}$ instead of $L$ ). These include using effective photon arrival PDFs from averaged stochastic tracks instead of minimally ionizing tracks, including non-uniform photo-multiplier noise modeling, removing photons that might arise from large stochastic losses, and convolving the firstorder statistic PDF with an energy-dependent Gaussian kernel, all of which are described in more detail in [26]. We call this ensemble of modifications "max settings" in the following. The general reconstruction scheme with B-splines as described above is referred to as SplineReco. When no added information is given, SplineReco uses likelihood $L_{1 \mathrm{st}}$ and the previously described max settings. As can be seen in figure 3 , usage of likelihood $L_{1 \text { st }}$ is better than the standard likelihood $(L)$ and the gamma distribution-based approach. The use of the modifications we refer to as max settings gives some additional improvement. Compared to the analytic gamma approximation, the PDFs based on B-splines are significantly more precise and still reasonably fast. A typical application of the reconstruction on a $10 \mathrm{TeV}$ muon takes less than a hundredth of a second. A more thorough comparison of running times is given in section 4.3. While the modifications have been empirically shown to somewhat circumvent the underlying unphysical assumption of an infinite muon track with a smooth energy loss profile, it is preferable to model a more correct hypothesis. The new algorithm that models stochastic losses directly is described in section 3 .

\subsection{Uncertainty estimation}

The previously discussed likelihood optimizations typically run over 6 parameters: $\Theta=(x, y, z, t$, $\theta, \phi)$. The first four define a point lying on the track $(x, y, z, t)$ and the last two specify the direction using zenith and azimuth $(\theta, \phi)$. The angle parameters are often re-parametrized for technical reasons (see appendix A for details). In order to obtain an uncertainty estimate for the two angles, the typical practice is to fit a 2-D paraboloid to the profile-likelihood at the minimum [17], where the $x, y, z$ and $t$ parameters are profiled, i.e. optimized for each value of the two angles. This uncertainty estimation is referred to as the traditional method in the following. In practice, a grid is constructed in a rotated coordinate system $\varphi_{1}, \varphi_{2}$, which is localized at the equator such that the two new angle coordinates are comparable [17] (see appendix A.2 for more information). A problem with this construction is that the fixed grid used for the evaluation of the likelihood space is optimized for a typical uncertainty of about a degree. If the actual uncertainty is much smaller or larger than this typical uncertainty, this can lead to failures on the paraboloid fit. Additionally, if a fit converges, it is not clear how well it describes the shape of the log-likelihood maximum. Especially at lower energies that shape is typically not parabolic. In section 3 an updated strategy is described that avoids both the problem with the fixed grid and the fit quality check.

\section{New algorithm: SegmentedSplineReco}

At energies above $\sim 1 \mathrm{TeV}$, muons predominantly lose energy stochastically via bremsstrahlung, pair production, and nuclear interactions [15]. Therefore, the assumption of a continuous energy 


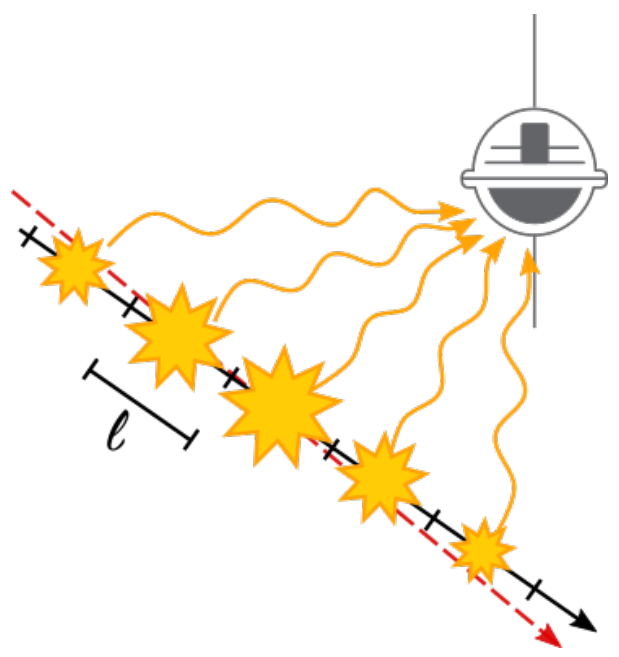

Figure 4. Schematic view of SegmentedSplineReco. The incoming muon track in red is first reconstructed by the black line, representing the initial track hypothesis for SegmentedSplineReco. It follows an energy reconstruction which results in a series of cascades along the muon track (yellow stars), placed at the center of each segment of length $\ell$. The energy information of each segment is used to define the final PDF at each DOM using equation (3.1).

loss pattern used in the reconstructions presented up to now is no longer valid. The result of these stochastic energy losses is the production of clustered light depositions on top of the track signature. Light created in such stochastic losses has a different emission spectrum, and it influences the photon arrival time distribution. Therefore, these stochastic energy losses should ideally be included directly in the track parametrization. A new reconstruction implementing this idea is described in this section. This reconstruction is referred to as SegmentedSplineReco in the following.

\subsection{Angular reconstruction}

SegmentedSplineReco is a maximum likelihood reconstruction that uses a segmented muon hypothesis (figure 4). Each segment effectively models electromagnetic and hadronic stochastic losses ("cascades"), and contributes to the PDF of the photon arrival times, together with a constant DOM-dependent noise term and an optional infinite minimum-ionizing muon track hypothesis. The number of photons and their time arrival distributions are obtained from high-dimensional splines fitted to Monte Carlo simulations of photons propagating in ice. Similar splines for the infinite muon hypothesis are used in the SplineReco reconstruction. The main difference are the additional B-splines for the stochastic losses in SegmentedSplineReco.

The reconstruction performs several steps which are described below:

1. The initial hypothesis is twofold: (1) a track direction and (2) an energy loss pattern parametrized by electromagnetic cascades placed at the center of each segment and located along the initial track hypothesis (see figure 4). These first guesses are given by previous reconstructions. Alternatively the energy loss pattern can also be determined directly by SegmentedSplineReco, in which case only an initial guess of the track direction is required. 


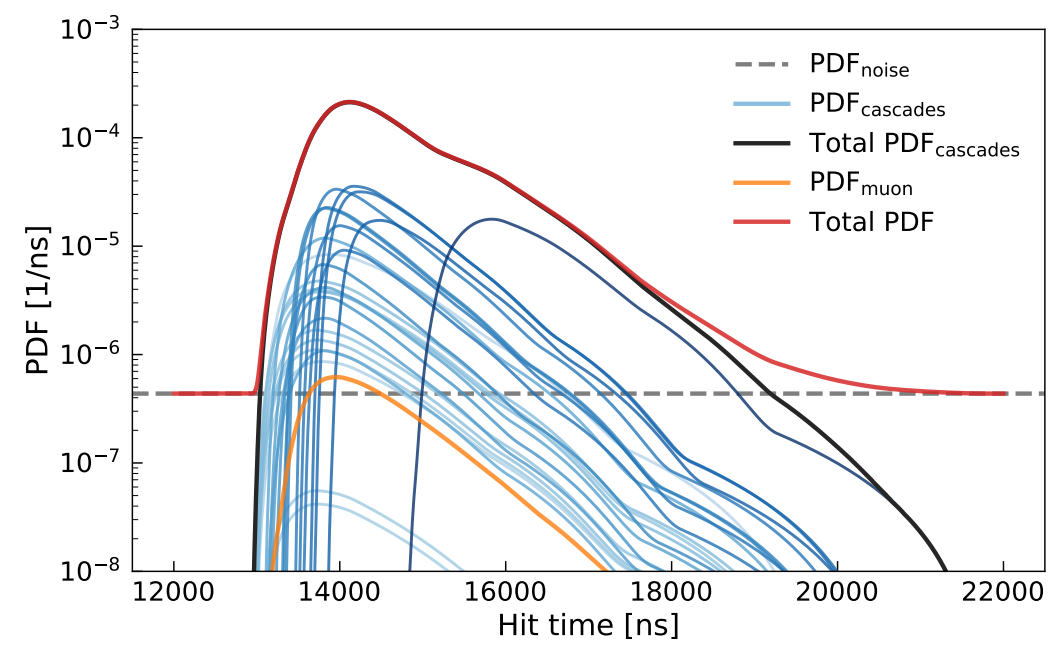

Figure 5. SegmentedSplineReco PDF for one DOM as a function of the photons arrival time, according to equation (3.1). The blue lines are the PDFs of each cascade along the muon track in order of creation from lighter to darker blue; their weighted sum is given by the solid black line. The orange line shows the PDF for a minimum-ionizing muon, the dashed gray line the noise PDF. The total PDF is shown by the red curve.

2. The total PDF of the photon arrival time $t$ at a DOM position in the detector is given by the weighted sum of $n$ PDFs using each cascade, noise and potentially a minimum-ionizing muon as a source of photon emission:

$$
p(t)=\sum_{k=0}^{n} w_{k} p_{k}(t),
$$

where $n=N_{\text {casc. }}+1^{5}, N_{\text {casc. }}$ denotes the number of shower segments and $w_{j}=\frac{\lambda_{j}}{\sum_{k=1}^{n} \lambda_{k}}$. The parameter $\lambda_{j}$ denotes the expected number of photons of source $j$ in the given DOM, where the different sources are the electromagnetic cascades, the constant noise contribution, and the contribution of a minimum-ionizing muon if requested. The total number of photons from the cascades and the muon are again obtained from high-dimensional spline distributions fitted to simulations. Figure 5 shows the PDF for all cascades (blue curves) produced along a muon track as a function of hit time, their weighted sum (black curve) and the infinite muon (orange curve) and noise (dashed gray line) PDFs.

3. The PDF is then used to define a likelihood function, which is maximized varying the track parameters $\Theta=(x, y, z, t, \theta, \phi){ }^{6}$ Three likelihood functions have been implemented:

(a) standard unbinned likelihood

$$
L(\Theta)=\prod_{j}^{N_{\mathrm{DOM}}} \prod_{i}^{N_{\mathrm{hit}}}\left[p_{j}\left(t_{i}\right)\right]^{q_{i}},
$$

\footnotetext{
${ }^{5}$ In the case we also fit the minimum-ionizing muon, we have $n=N_{\text {casc. }}+2$.

${ }^{6}$ Here we use the parameters $\theta$ and $\phi$, the usual angle parameters in spherical coordinates. In a more practical optimization these variables are re-parametrized in one of two different ways and then denoted either as $\varphi_{1}$ and $\varphi_{2}$ or $\Phi_{1}$ and $\Phi_{2}$ (see appendix A).
} 
(b) extended unbinned likelihood

$$
L_{\mathrm{ext}}(\Theta)=\prod_{j}^{N_{\mathrm{DOM}}} \frac{e^{-\lambda_{j}} \lambda_{j} q_{j}}{q_{j} !} \prod_{i}^{N_{\mathrm{hit}}}\left[p_{j}\left(t_{i}\right)\right]^{q_{i}},
$$

(c) unbinned likelihood for the first hit on each DOM:

$$
L_{1 \mathrm{st}}(\Theta)=\prod_{j}^{N_{\mathrm{DOM}}} p_{j, 1 \mathrm{st}}\left(t_{1}\right) .
$$

The index $j$ runs over all DOMs while the index $i$ runs over all hits for a given DOM $j$. The total charge produced by a hit $i$ is denoted by $q_{i}$. The PDF $p_{j, 1}$ in likelihood (c) is the first-order-statistic PDF, this time derived from the total PDF of all source contributions. The derivation is mathematically similar to the one for a single minimal-ionizing muon $L_{1 \mathrm{st}}$ in equation (2.2).

SegmentedSplineReco has been implemented in C++ and Python within the IceCube software framework. It includes several improvements with respect to the previous algorithms. This includes support for exact gradient and calculation of the second-order partial derivatives matrix, the Hessian matrix, from the underlying high-dimensional B-splines and the possibility to fit the energies jointly with the track parameters. The latter option is only feasible with available gradient information due to the rather high-dimensional (> 100-D) problem. Supplying the optimization algorithm with an exact gradient leads to substantially improved convergence speed. For the often used algorithm MIGRAD contained within the high-energy physics package minuit [27] the resulting increase in speed is a factor of two compared to not using a gradient.

A new coordinate system has also been implemented, equivalent to the one described in section 2.2: the seed track is rotated to the equator of the coordinate system, defining a new origin. The coordinates near the equator are quasi-euclidean for small values and remain interpretable as angles for larger values. More details can be found in appendix A.

Finally, an energy-dependent convolution of the first-order statistic PDF has been implemented similar to the implementation in SplineReco. The SegmentedSplineReco likelihood is convoluted with a Gaussian distribution using a fast recursive approximation algorithm as implemented in [28]. Such a convolution implicitly models timing inaccuracies between modules and also mitigates remaining model mis-specification. Some of these inaccuracies include the data acquisition uncertainty $(\sim 0.7 \mathrm{~ns})$, due to the signal transmission times [1], and the geometrical uncertainty $(\sim 1 \mathrm{~ns})$ due to uncertainty in the position of the DOMs [2]. The likelihood is calculated at several sample points around the requested time. From these sample points, the convoluted PDF is calculated with adjustable accuracy depending on sample point density and recursion step count. The convolution is sometimes called post-jitter below.

\subsection{Uncertainty estimation}

Two new uncertainty estimation methods have been implemented for SegmentedSplineReco. Both of them estimate the Hessian matrix at the log-likelihood optimum. The first approach (Method 1) 
calculates it analytically using the ability of the B-spline PDFs to yield exact higher-order derivatives. The second approach (Method 2) samples the 6-D minimum of the negative log-likelihood function in the track parameters $\left(x, y, z, t, \varphi_{1}, \varphi_{2}\right)^{7}$ with a Markov Chain Monte Carlo (MCMC) sampler. The result is used to fit a 6-D elliptic paraboloid to the log-likelihood landscape which again requires the calculation of the $6 \times 6$ Hessian matrix. Details are given in appendix B. While this approach is more time consuming, it can be a little more robust against non-Gaussianities close to the optimum if a modified $\chi^{2}$ loss function is used (see appendix B) and often leads to slightly wider contours as shown in section 4.

The inverse of the obtained Hessian yields the covariance matrix containing the parameter correlations. A reduction to the $2 \times 2$ submatrix of the angle parameters marginalizes the other parameters and results in a 2-D uncertainty ellipse for the direction. In comparison, the traditional method described in section 2.2 performs a paraboloid fit using profile likelihood evaluations in the two angular dimensions. Performing these profile likelihood evaluations is time consuming and can lead to unstable results, while the fixed grid size makes it unadaptable to different uncertainty angular scales. Both of these problems are solved with either of the new approaches. In particular, the new methods can detect when the outcome of the uncertainty estimation is unreliable as explained in appendix B.

Another possibility that comes with the analytic Hessian (Method 1) is to jointly calculate the Hessian with respect to the six track parameters and additionally the energy parameters of all individual energy losses. Computationally, this full calculation has nearly no overhead, but it broadens the final uncertainty contours over the two angular dimensions due to the extra marginalization over the energy dimensions if it is used. This can be desirable, since the uncertainty is by construction too small if the energies of stochastic losses are fixed (see section 4.2). However, if the energies are fixed in the previous optimization procedure, and also due to the high dimensionality of the problem, the enlarged Hessian is usually not calculated at a local minimum and often not positive definite, so we do not use this procedure in practice.

\section{Performance comparisons}

The new reconstruction has been applied to three different selections of simulated muon track events. They have been selected in order to have a complete representation of the typologies of events entering in various IceCube selections. The first dataset contains muon tracks that pass quality cuts (so-called NDir/LDir cuts ${ }^{8}$ on the number of hit DOMs and track length, respectively; see [12] for more information) based on SplineReco, which to some extent mimics events that are usually found on the final analysis selections used in IceCube. In the following they are referred to as SplineReco-optimized. Events with large stochastic losses typically obtain low NDir/LDir values with SplineReco. These events subsequently do not pass the cuts and should be mostly absent in this selection. Nevertheless, it is instructive to see whether SegmentedSplineReco can give improvements also for these events. The other two datasets are based on a geometrical Monte Carlobased selection. One contains muon tracks starting outside the detector volume with a minimal track length of $700 \mathrm{~m}$ (Through-going events), the other muon tracks that start in the detector volume and have a minimal track length of $400 \mathrm{~m}$ (Starting events). Starting events are expected to give the

\footnotetext{
${ }^{7}$ The angles here are defined in the rotated parametrization (see appendix A).

${ }^{8}$ The exact cuts are LDir $\geq 600$ and NDir $\geq 8$.
} 

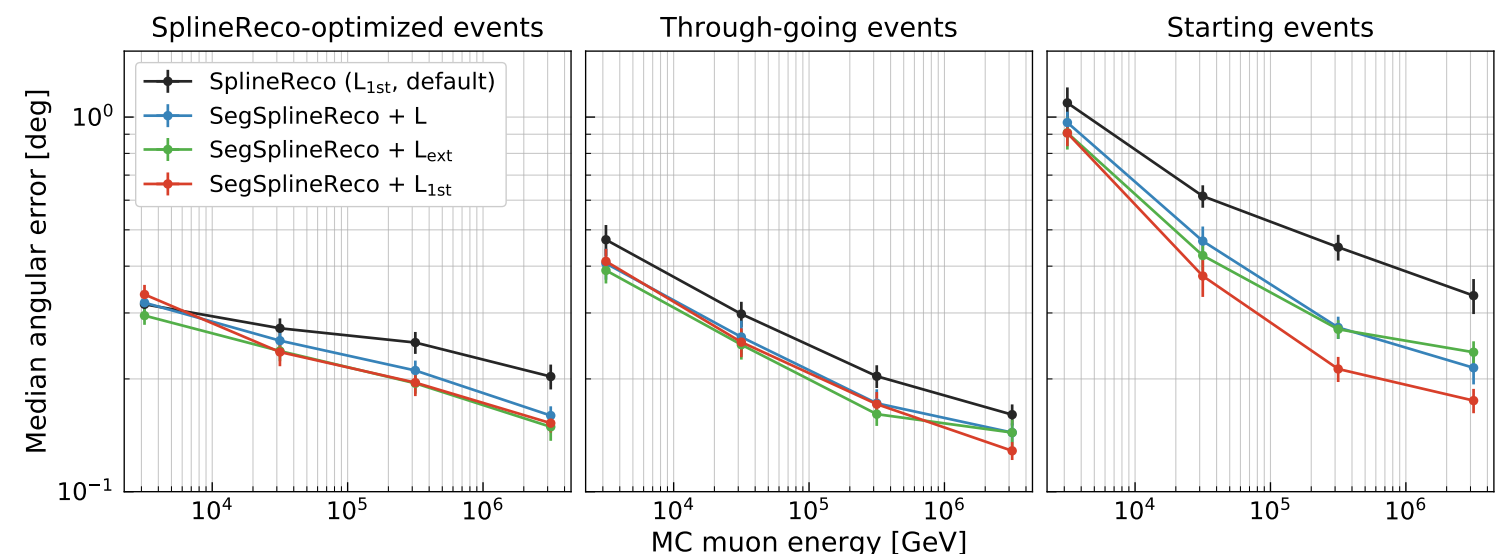

Figure 6. Median angular resolution as a function of MC muon energy calculated at the interaction vertex for three IceCube all-sky simulations: SplineReco-optimized events, through-going and starting tracks. The SegmentedSplineReco reconstruction is compared to SplineReco (black line). The three different likelihood models for SegmentedSplineReco are compared: the standard unbinned likelihood $L$ (blue line), the extended unbinned likelihood $L_{\text {ext }}$ (green line) and the unbinned likelihood for the first hit per DOM $L_{1 \text { st }}$ (red line). The statistical error on the median is calculated using bootstrapping.

largest improvements with the new reconstruction since they start with a cascade inside the detector, which was not taken into account during reconstruction in SplineReco.

\subsection{Angular resolution}

To illustrate the performance of the new reconstruction, figure 6 shows the median angular difference between true muon direction and reconstructed muon direction for the three classes of track-like events and the three likelihood formulations. These are compared to SplineReco default settings, i.e. without any of the modifications (see section 2 for a description of these modifications). It can be seen that SegmentedSplineReco yields up to $20 \%$ better angular resolutions at high energies for through-going tracks, and up to a factor 2 better resolutions for starting tracks. The likelihood that looks only at the first hit $\left(L_{1 \mathrm{st}}\right)$ performs generally as good or better than the other two, which is the known behavior that is observed for the prevailing reconstructions (section 2) and understood as mitigation of the unphysical hypothesis and the uncertainties of the ice model. The similarity of all likelihoods for through-going tracks indicates that the stochastic modelling in SegmentedSplineReco improves the overall data description and narrows the advantage of $L_{1 \mathrm{st}}$, even though the slight difference in outcomes shows that the modelling is not perfect. The difference in outcomes is to be compared with the respective difference for SplineReco (compare SplineReco + $L$ and SplineReco $+L_{1 \mathrm{st}}$ in figure 3), which is much larger. For starting tracks, a clear advantage for $L_{1 \mathrm{st}}$ remains. A potential explanation for this behavior is that the seed reconstruction used for these tracks is often skewed and the pure energy fit to determine a fixed energy loss profile subsequently gives a result that is rather far from the true energy loss profile. In these cases, $L_{1 \mathrm{st}}$ best manages the resulting inaccuracies on the model.

In figure 6, the initial energy loss pattern is obtained by performing a pure energy fit with SegmentedSplineReco. This energy profile is then used as seed for the track reconstruction. Seg- 
mentedSplineReco can also perform a simultaneous fit of track parameters and energy cascades. However, this second option gives worse angular resolution, as shown for $L_{\text {ext }}$ with joint vertex and energy optimization in figure 7. The worse performance is probably due to numerical instability issues of the high-dimensional problem. For this reason, by default the energies are always determined independently before the vertex parameters are optimized.

Figure 7 also shows how SegmentedSplineReco with $L_{1 \text { st }}$ compares to SplineReco with ("max") and without modifications ("default"). One of those modifications is an energy-dependent convolution of the time PDF of the first hit (post-jitter), which models absolute time detection uncertainty between DOMs. Since SegmentedSplineReco improves the angular resolution only at the highest energy when compared to SplineReco with max settings, this convolution has also been applied to the new reconstruction. As shown in the figure, besides an energy-dependent convolution, also a fixed-time resolution convolution has been implemented for three different post-jitter times: $2 \mathrm{~ns}$, $2.5 \mathrm{~ns}$ and $4 \mathrm{~ns}$. However, the energy-dependent post-jitter convolution improves the resolution at all energies. Therefore, the energy-dependent convolution is used as default setting in SegmentedSplineReco. If no energy estimator is available, a $4.5 \mathrm{~ns}$ convolution gives almost comparable results. Other modifications are not really applicable to SegmentedSplineReco, like the effective stochastic loss profile, since they are already naturally captured in the explicit stochastic modelling within SegmentedSplineReco. It can be seen in figure 8 that standard SegmentedSplineReco is on par or slightly better than SplineReco with these settings. The extra energy-dependent time PDF convolution is further improving the resolution by a few percent in all datasets.

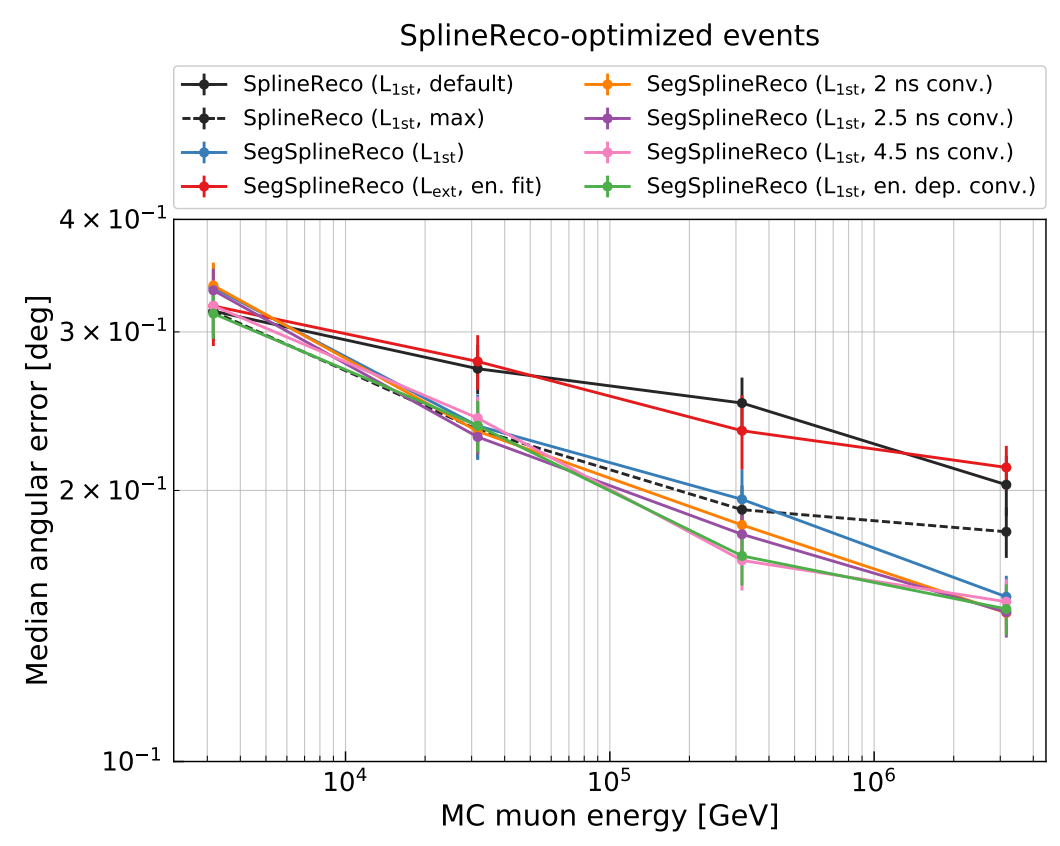

Figure 7. Median angular resolution as a function of MC muon energy calculated at the interaction vertex for the SplineReco-optimized events. The SplineReco with default (full line) and max settings (dashed line) are shown in black. The standard SegmentedSplineReco (blue line) is compared with SegmentedSplineReco $\left(L_{\text {ext }}\right)$ with jointly fitted energy cascades (red line). The SegmentedSplineReco reconstruction using $L_{1 \mathrm{st}}$ with energy-dependent PDF convolution is compared with a fixed convolution of $2 \mathrm{~ns}, 2.5 \mathrm{~ns}$ and $4.5 \mathrm{~ns}$, each modeling a different assumption of absolute time detection uncertainty between DOMs. 

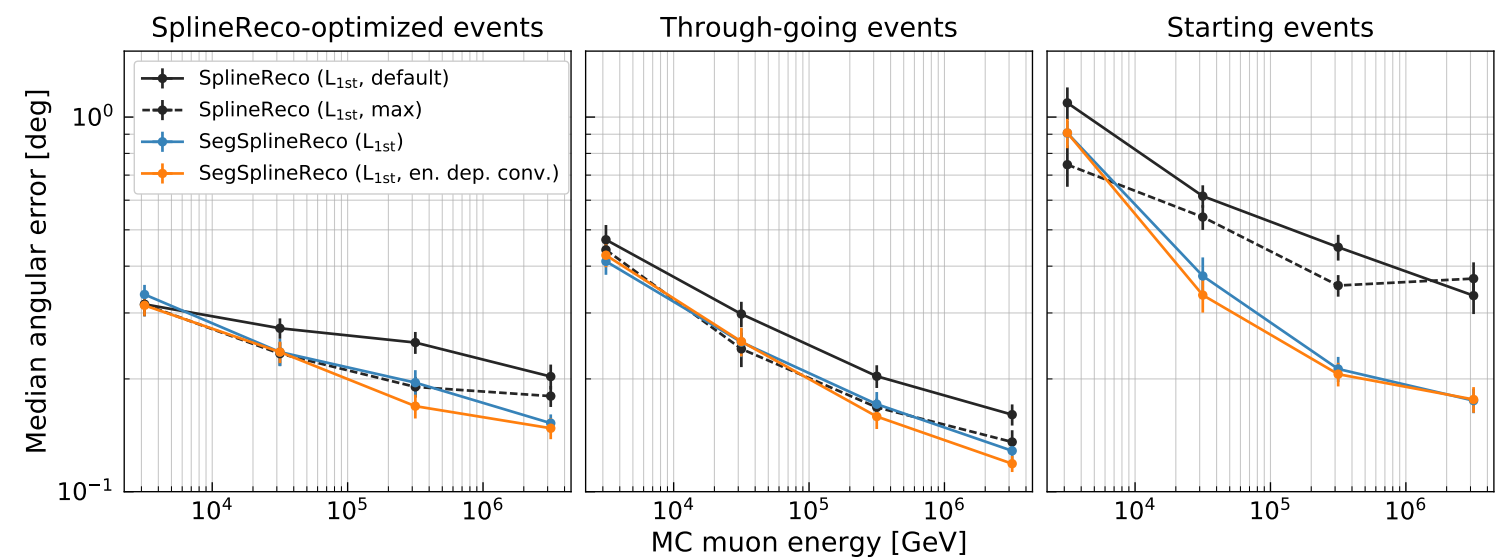

Figure 8. Median angular resolution as a function of MC muon energy calculated at the neutrino interaction point for three IceCube all-sky simulations: SplineReco-optimized events, through-going and starting tracks. The SegmentedSplineReco reconstruction using the $L_{1 \text { st }}$ likelihood with (orange line) and without (blue line) energy-dependent convolution (post-jitter) is compared to SplineReco with default settings (solid black line) and max settings (dashed black line). The statistical error on the median is calculated using bootstrapping.

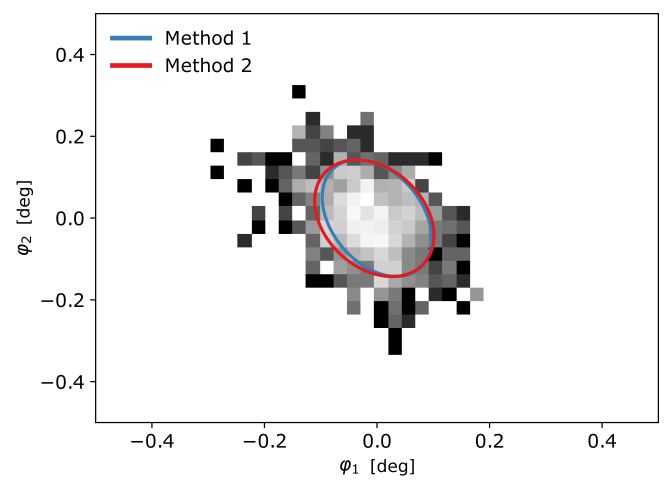

(a) Event with Gaussian minimum.

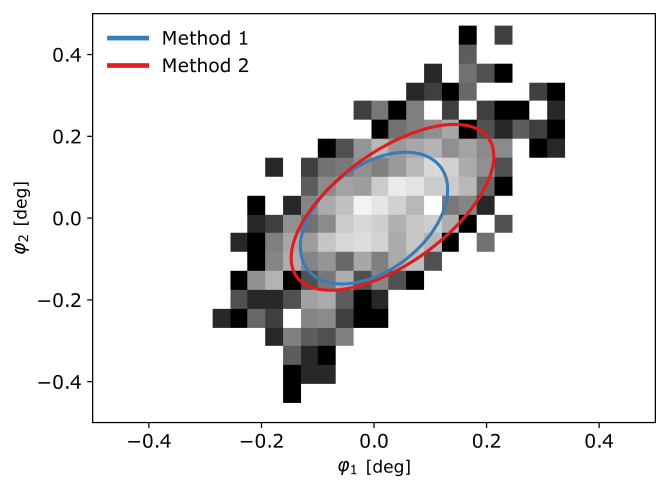

(b) Event with slightly non-Gaussian minimum.

Figure 9. Comparison of both uncertainty calculations for two example events. The two $68 \%$ uncertainty ellipses are shown on top of the MCMC samples, which indicate the true marginalized density assuming a flat prior.

\subsection{Uncertainty estimation}

As discussed in section 3.2, two methods for the calculation of uncertainty contours are implemented in the new reconstruction. Figure 9 shows the uncertainty contours for two example events. Additionally, the marginalized PDF from the MCMC samples is indicated assuming a flat prior. In general, the uncertainty contour from the analytic Hessian (Method 1) is smaller than the paraboloid fit (Method 2) for all events that show some non-Gaussian behavior. The quality of the uncertainty estimation can be judged by its coverage.

For simplicity we average major and minor axes of the uncertainty contour to create an averaged angular error for $\Delta \Psi$, the difference between reconstructed direction and true direction. 

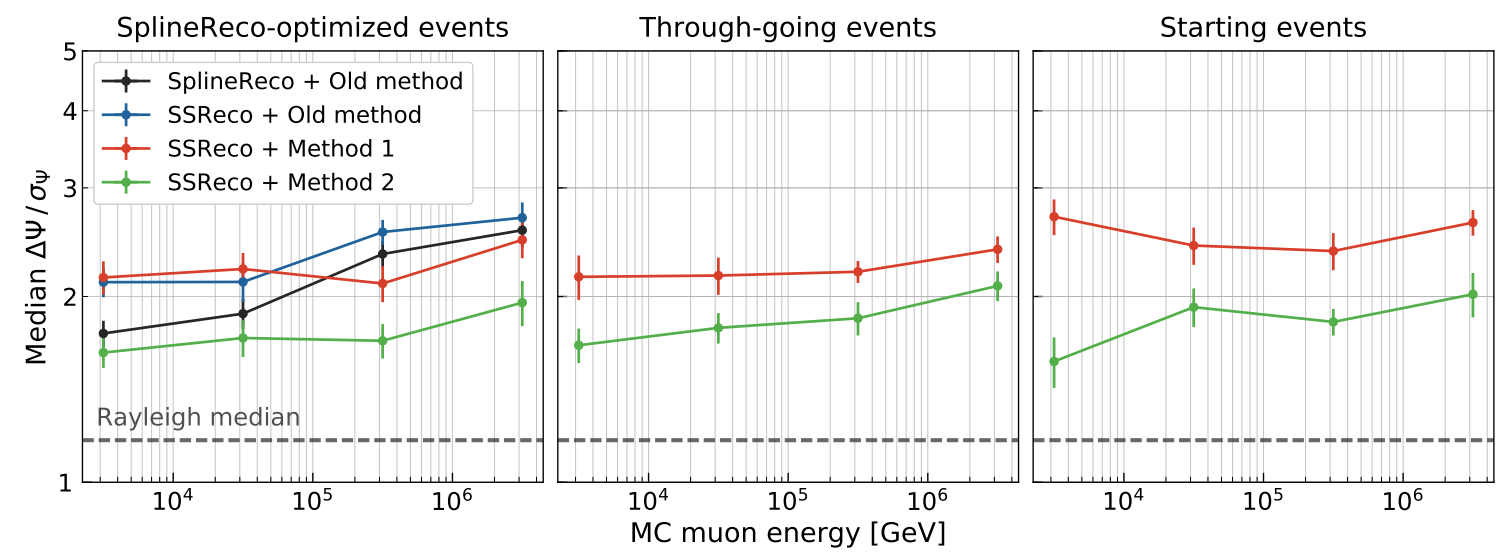

Figure 10. Comparison of different angular error estimators for the SegmentedSplineReco reconstruction with likelihood model (c) of the SplineReco-optimized, through-going and starting events. The median of the pull $\left(\Delta \Psi / \sigma_{\Psi}\right)$ is shown as a function of the MC muon energy. The statistical error on the median is calculated using bootstrapping. The median angular resolution $\Delta \Psi$ is obtained using the $L_{1 \text { st }}$ likelihood with energy-dependent post-jitter. $\sigma_{\Psi}$ has been calculated for the same muon track obtained from likelihood $L_{1 \text { st }}$ without energy-dependent post-jitter. The dashed gray line shows the ideal case of a Rayleigh distribution with $\sigma=1$ and median at 1.17. The MC muon energy is calculated at the neutrino interaction point.

This averaged uncertainty is calculated using $\sigma_{\Psi}=\sqrt{\frac{\sigma_{1}^{2}+\sigma_{2}^{2}}{2}}$. The quantity $\Delta \Psi / \sigma_{\Psi}$ is called the pull. Ideally, it is distributed like a Rayleigh distribution with $\sigma=1$ which has a median of 1.17. Figure 10 shows the median pull for the three types of events. It compares both uncertainty calculations, and also includes the old 2-D paraboloid fit [17] presented in section 2.2 for the SplineReco-optimized event class. It can be seen that the pull is generally flatter with the new uncertainty estimation, which shows that the shortcomings of fixed grid size and instability from profiling in the old uncertainty estimation are gone. However, a slight energy dependence remains, which seems to increase at the highest energy. This is reflected in the overall offset between the two curves in the median pull. Overall, the median is larger than the ideal value of 1.17 in any approach, which shows that the likelihood contours are underestimating the true uncertainty and further systematic effects are present. The underestimation partly stems from the fact that the energies of the stochastic losses are fixed. Additional contributions likely come from remaining model mis-specifications, like the assumption that all energy losses are modelled as point-like electromagnetic cascades at fixed distances along the track and from non-Gaussian behavior around the local optima.

\subsection{Runtime}

Table 1 shows a comparison of the running time for SplineReco and SegmentedSplineReco with the corresponding uncertainty estimations for different energy ranges. The reconstruction times are the average CPU time for 100 standard likelihood evaluations, expressed in minutes. Each reconstruction has been performed on the SplineReco-quality dataset. The new reconstruction is significantly more time consuming.

On average, the running time per event for SegmentedSplineReco that includes a standard vertex fit and the error estimation with Method 1 is of $\sim 1.25$ minutes, about 6 times larger than 
the running time required to perform a SplineReco vertex fit and uncertainty estimation with the traditional method. This run time fraction doubles when also the energy fit is performed for SegmentedSplineReco and can get up to 100 times larger when performing the energy-dependent post-jitter convolution and using Method 2 for the uncertainty estimation.

Table 1. Running time comparison in minutes of some relevant reconstructions and uncertainty estimations. The reconstruction times are the average CPU time for 100 standard likelihood evaluations to give roughly comparable times that are independent of optimization algorithms. For SegmentedSplineReco the distance between two modelled energy losses is $10 \mathrm{~m}$.

\begin{tabular}{|c|c|c|c|c|c|c|c|}
\hline & \multicolumn{2}{|c|}{ SPlineReco } & \multicolumn{5}{|c|}{ SEgmentedSplineReco } \\
\hline & \multirow{2}{*}{$\begin{array}{c}\text { ReCOnStRUCtion } \\
\text { Vertex fit }\left(L_{1 \mathrm{st}}, \text { max settings }\right) \\
(100 \text { evals })\end{array}$} & \multirow{2}{*}{$\begin{array}{c}\text { UNCERTAINTY ESTIMATION } \\
\text { Traditional Method }\end{array}$} & \multicolumn{3}{|c|}{ RECONSTRUCTION } & \multicolumn{2}{|c|}{ UNCERTAINTY ESTIMATION } \\
\hline & & & $\begin{array}{l}\text { Energy fit } \\
\text { (full) }\end{array}$ & $\begin{array}{c}\text { Vertex fit } \\
(100 \text { evals })\end{array}$ & $\begin{array}{l}\text { With post-jitter } \\
\text { (100 evals) }\end{array}$ & Method 1 & Method 2 \\
\hline $1 \mathrm{TeV}-10 \mathrm{TeV}$ & 0.002 & 0.06 & 0.70 & 0.28 & 2.49 & 0.09 & 4.41 \\
\hline $10 \mathrm{TeV}-100 \mathrm{TeV}$ & 0.005 & 0.12 & 0.98 & 0.58 & 5.25 & 0.19 & 9.09 \\
\hline $100 \mathrm{TeV}-1 \mathrm{PeV}$ & 0.010 & 0.22 & 1.46 & 1.20 & 10.29 & 0.32 & 16.38 \\
\hline $1 \mathrm{PeV}-10 \mathrm{PeV}$ & 0.017 & 0.34 & 2.22 & 1.88 & 19.32 & 0.47 & 24.85 \\
\hline
\end{tabular}

\section{Discussion and outlook}

We have introduced a new directional reconstruction for muons that explicitly models the stochastic losses in the likelihood by equidistant electromagnetic showers along the track. For all track topologies and energies, the new reconstructions shows a better muon angular resolution. The improvement increases with energy as the stochastic modelling of the muon becomes more important. For throughgoing tracks the improvement is up to $10--20 \%$ at PeV energies. For starting tracks, it is much more pronounced and larger than a factor of 2 above $100 \mathrm{TeV}$. In addition to the reconstruction, the uncertainty estimation has been improved by a more numerically stable determination of the Hessian at the optimum. The resulting pull distribution is now nearly flat with energy using a likelihood that only looks at the first hit, which is a common procedure to mitigate systematics. While the new reconstruction is more precise it is also more time consuming. Depending on the settings, the reconstruction including uncertainty estimation takes about 6 (using no energy-dependent convolution and Hessian Method 1) to over 100 times more processing time (using energy-dependent convolution and Hessian Method 2) per event than the previously state-of-the-art muon reconstruction SplineReco. In later stages in typical event selection chains these processing times can still be feasible. A few limiting factors remain. Currently the stochastic losses are modeled by pointlike electromagentic showers at fixed distances from each other. In reality, these losses have longitudinal emission profiles and are not equi-distant. In particular if the track passes close to a DOM such an unphysical hypothesis can bias the reconstruction. However, it is hard to see how such an assumption can be made more realistic in the parametric likelihood approach. A remaining drawback that can potentially be fixed is the currently neglected correlation between the true muon energy and the energy losses. Fitting simultaneously for the muon energy could stabilize and improve the energy loss solution. We leave that for future work.

Systematic uncertainties have not been included in this work. Some of the sources of systematics are known, such as the scattering and absorption coefficients of the glacial ice. However, the effect of these systematic uncertainties can only be studied in dedicated simulations by varying these 


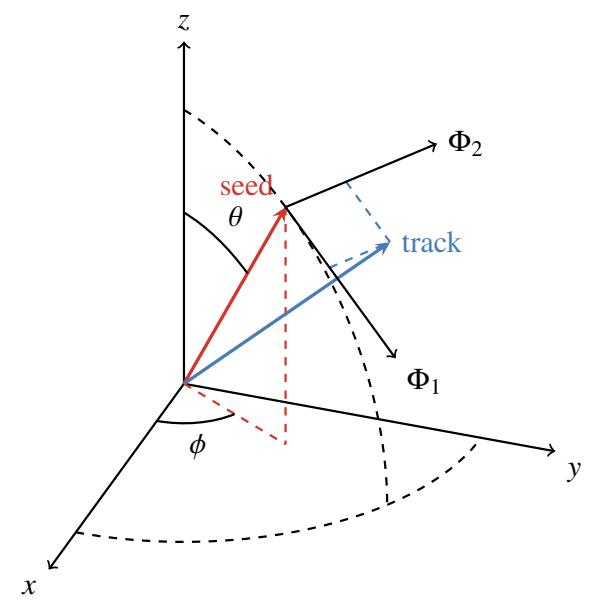

Figure 11. The auxiliary angular coordinates behave as Euclidean coordinates $\Phi_{1}$ and $\Phi_{2}$ in a unique tangential plane defined by the seed track.

parameters. We also did not study the performance on real data, which to some extent can be studied with the moon shadow. Efforts in both these directions are currently ongoing in IceCube and we leave these studies for future publications.

\section{A Parametrizations of track orientations}

Instead of zenith $\theta$ and azimuth $\phi$, it is more stable to change the parametrization of the angles to auxiliary coordinates that are independent of the position on the sphere. This is the case for both the optimization and uncertainty estimation procedures. Two common re-parametrizations are described in the following.

\section{A.1 Tangent plane parametrization}

At the current seed direction the tangent plane defines a new coordinate system which replaces $\theta$ and $\phi$ (see figure 11). The coordinate axes are uniquely defined by a choice of orthogonal axes with respect to the seed track $\vec{s}=\left(s_{x}, s_{y}, s_{z}\right)$. An example choice is $\vec{e}_{\Phi_{1}}=\left(0, \frac{s_{x}}{n},-\frac{s_{y}}{n}\right)$, and $\vec{e}_{\Phi_{2}}=\left(-n, \frac{s_{x} \cdot s_{y}}{n}, \frac{s_{x} \cdot s_{z}}{n}\right)$ with $n=\sqrt{s_{y}^{2}+s_{z}^{2}}$. The current track hypothesis is located at $\left(\Phi_{1}, \Phi_{2}\right)$, where $\Phi_{1}$ and $\Phi_{2}$ are new optimization parameters. A drawback of this scheme is that for large deviations from the seed the parameters lose the meaning of an angle. This can be undesired behavior if uncertainty estimation is performed in these coordinates. The tangent plane parametrization is often used by the prevailing reconstructions.

\section{A.2 Rotation to $(1,0,0)$}

This parametrization is defined by the unique rotation $R$ of the current seed position to the $x$-axis (see figure 12). The auxiliary angles $\varphi_{1}$ and $\varphi_{2}$ measure the rotated track coordinates relative to the rotated seed which aligns with the $x$-axis. The inverse rotation $R^{-1}$ applied to the current rotated track yields back the track orientation in the original coordinate system. An advantage of this parametrization is that $\varphi_{1}$ and $\varphi_{2}$ are always defined as angles and it is therefore suited for 


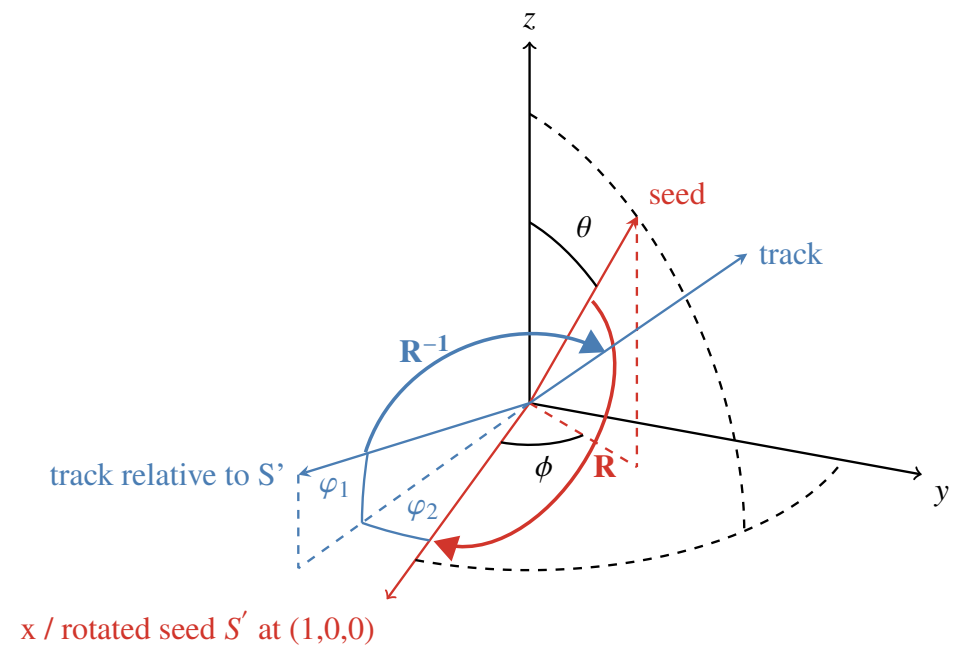

Figure 12. The auxiliary angular coordinates $\varphi_{1}$ and $\varphi_{2}$ are measured with respect to the rotated Seed track $S^{\prime}$ that aligns with the x-axis. They behave as standard angular coordinates.

uncertainty contours that can measure tens of degrees in diameter. The parametrization is used by the prevailing uncertainty estimation precedure [17]. It is also used by the new reconstruction SegmentedSplineReco and the subsequent uncertainty estimation.

\section{B Technical details of the uncertainty estimation}

This section describes the technical details of the uncertainty estimations introduced in section 3.2. From Wilks' theorem [29] it follows that the quantity $\lambda=2 \cdot\left(\log \mathrm{L}(\theta)-\log \mathrm{L}\left(\theta_{0}\right)\right)$ is approximately $\chi_{n}^{2}$-distributed with $n$ degrees of freedom if $n$ is the number of nested parameters. At the same time, the $\chi_{n}^{2}$-distribution is equivalent to the logarithmic difference of the $n$-dimensional Multivariate Gaussian distribution at its maximum with any other point drawn from the Gaussian [30]. Therefore, $\lambda$ can be approximated around $\theta_{0}$ with

$$
\begin{aligned}
\lambda & =-2 \cdot\left(\log \mathrm{L}(\theta)-\log \mathrm{L}\left(\theta_{0}\right)\right) \\
& \approx\left(\theta-\theta_{0}\right) \cdot \operatorname{Cov}_{\mathrm{n}}{ }^{-1} \cdot\left(\theta-\theta_{0}\right) \\
& =\left(\theta-\theta_{0}\right) \cdot H_{0} \cdot\left(\theta-\theta_{0}\right)
\end{aligned}
$$

where $\operatorname{Cov}_{\mathrm{n}}{ }^{-1}$ is the inverse covariance matrix of the related n-dimensional Gaussian, which simultaneously is equivalent to the Hessian matrix $H_{0}$ of the log-likelihood function at the optimum $\theta_{0}$. We can therefore write for $-\log \mathrm{L}(\theta)$ close to the optimum $\theta_{0}$

$$
\begin{aligned}
-\log L(\theta) & \approx\left(\theta-\theta_{0}\right) \cdot \frac{H_{0}}{2} \cdot\left(\theta-\theta_{0}\right)-\log L\left(\theta_{0}\right) \\
& \equiv(\theta-A) \cdot \frac{B}{2} \cdot(\theta-A)+C \\
& =f(\theta ; A, B, C)
\end{aligned}
$$

which is the formula for a general elliptic paraboloid in $n$ dimensions with position A, parameter matrix $\mathrm{B} / 2$ and offset $\mathrm{C}$. With these relations it is clear that determination of $H_{0}$ allows to determine 
the covariance matrix, and thereby Gaussian contours, after matrix inversion. As discussed in section 3.2, two methodologies to estimate $H_{0}$ have been developed and are described in the following.

Method 1. The first method calculates the Hessian matrix at the optimum, $H_{0}$, analytically. Advances in automatic differentiation have only recently made this feasible, in particular we used autograd $^{9}$ to crosscheck the implementation.

Method 2. The second method fits a paraboloid to samples $\operatorname{logL}\left(\hat{\theta}_{i}\right)$ of the negative likelihood function near the optimum. The samples are obtained with an affine-invariant particle-based Markov-Chain sampler, emcee [31]. The particle positions are initialized as samples drawn from a Gaussian distribution with covariance $H_{0}^{-1}$, where $H_{0}$ is the analytically calculated Hessian at the optimum (Method 1). This initialization skips the burn-in phase completely if the loglikelihood optimum is nearly Gaussian, and otherwise drastically speeds up convergence. For the 6-dimensional sampling ( $x, y, z, t$ and two angle dimensions) a burn-in phase of 2000 evaluations (100 particles with 20 iterations) followed by another 2000 samples is usually enough. In the second step a Levenberg-Marquardt algorithm is used to minimize the loss function

$$
\text { Loss }=0.5 \cdot \sum_{i} \rho\left(\left[-\log \mathrm{L}\left(\theta_{i}\right)-f\left(\theta_{i} ; A, B, C\right)\right]^{2}\right)
$$

and thereby fit a paraboloid shape to the samples. The term $\rho(x)=x$ yields the standard least-square loss. Empirically an often more robust fit is achieved with $\rho(x)=2 \cdot(\sqrt{1+x}-1)$, which represents a "soft-11" distance that better handles non-Gaussianities or irregularities in the likelihood samples. ${ }^{10}$ The parameter $A$ represents an n-dimensional mean and $C$ represents a 1-dimensional offset. The $n \times n$ parameter matrix $B$ has to be positive definite. This is ensured by a parametrizion in terms of its lower-triangular Cholesky-decomposition, which involves $n$ strictly positive parameters on the diagonal and $\frac{n^{2}-n}{2}$ parameters for the lower-triangular off-diagonal elements. The total number of parameters of the least-square fit is then $\frac{n^{2}+n}{2}+n+1$. If the resulting mean $A$ is sufficiently different from $\theta_{0}$, or if the offset $C$ is sufficiently different from $-\operatorname{logL}\left(\theta_{0}\right)$, this can indicate strong non-Gaussian behavior that even a modified least-square fit can not handle. In general this method yields slightly wider and more conservative contours than the analytic calculation (see also figure 9), because nonGaussianities in combination with the soft least-square fit widen the tails of the paraboloid solution.

\section{Acknowledgments}

The IceCube collaboration acknowledges the significant contributions to this manuscript from Federica Bradascio and Thorsten Glüsenkamp. The authors gratefully acknowledge the support from the following agencies and institutions: U.S.A. - U.S. National Science Foundation-Office of Polar Programs, U.S. National Science Foundation-Physics Division, U.S. National Science Foundation-EPSCoR, Wisconsin Alumni Research Foundation, Center for High Throughput Computing (CHTC) at the University of Wisconsin-Madison, Open Science Grid (OSG), Extreme Science and Engineering Discovery Environment (XSEDE), Frontera computing project at the Texas

\footnotetext{
${ }^{9} \mathrm{https} / / /$ github.com/HIPS/autograd.

${ }^{10}$ See the documentation of Levenberg-Marquardt algorithm on the scipy webpage for more details: https://docs.scipy.org.
} 
Advanced Computing Center, U.S. Department of Energy-National Energy Research Scientific Computing Center, Particle astrophysics research computing center at the University of Maryland, Institute for Cyber-Enabled Research at Michigan State University, and Astroparticle physics computational facility at Marquette University; Belgium — Funds for Scientific Research (FRS-FNRS and FWO), FWO Odysseus and Big Science programmes, and Belgian Federal Science Policy Office (Belspo); Germany - Bundesministerium für Bildung und Forschung (BMBF), Deutsche Forschungsgemeinschaft (DFG), Helmholtz Alliance for Astroparticle Physics (HAP), Initiative and Networking Fund of the Helmholtz Association, Deutsches Elektronen Synchrotron (DESY), and High Performance Computing cluster of the RWTH Aachen; Sweden - Swedish Research Council, Swedish Polar Research Secretariat, Swedish National Infrastructure for Computing (SNIC), and Knut and Alice Wallenberg Foundation; Australia - Australian Research Council; Canada Natural Sciences and Engineering Research Council of Canada, Calcul Québec, Compute Ontario, Canada Foundation for Innovation, WestGrid, and Compute Canada; Denmark - Villum Fonden and Carlsberg Foundation; New Zealand — Marsden Fund; Japan — Japan Society for Promotion of Science (JSPS) and Institute for Global Prominent Research (IGPR) of Chiba University; Korea National Research Foundation of Korea (NRF); Switzerland - Swiss National Science Foundation (SNSF); United Kingdom — Department of Physics, University of Oxford.

\section{References}

[1] IceCube collaboration, The IceCube Data Acquisition System: Signal Capture, Digitization, and Timestamping, Nucl. Instrum. Meth. A 601 (2009) 294 [arXiv: 0810 . 4930].

[2] IceCube collaboration, The IceCube Neutrino Observatory: Instrumentation and Online Systems, 2017 JINST 12 P03012 [arXiv: 1612 . 05093].

[3] R.C. Bay, R.A. Rohde, P.B. Price and N.E. Bramall, South pole paleowind from automated synthesis of ice core records, J. Geophys. Res. 115 (2010) D14126.

[4] ICECube collaboration, South pole glacial climate reconstruction from multi-borehole laser particulate stratigraphy, J. Glaciol. 59 (2013) 1117.

[5] ICECube collaboration, Measurement of South Pole ice transparency with the IceCube LED calibration system, Nucl. Instrum. Meth. A 711 (2013) 73 [arXiv: 1301.5361].

[6] IceCube collaboration, The IceCube Neutrino Observatory Part VI: Ice Properties, Reconstruction and Future Developments, in 33rd International Cosmic Ray Conference, 9, 2013 [arXiv: 1309.7010].

[7] IceCube collaboration, Light diffusion in birefringent polycrystals and the IceCube ice anisotropy, PoS ICRC2019 (2020) 854 [arXiv: 1908 . 07608].

[8] ICECube collaboration, Observation of High-Energy Astrophysical Neutrinos in Three Years of IceCube Data, Phys. Rev. Lett. 113 (2014) 101101 [arXiv: 1405 . 5303].

[9] ICECube collaboration, Constraints on Galactic Neutrino Emission with Seven Years of IceCube Data, Astrophys. J. 849 (2017) 67 [arXiv: 1707.03416$].$

[10] ICECube collaboration, The contribution of Fermi-2LAC blazars to the diffuse TeV-PeV neutrino flux, Astrophys. J. 835 (2017) 45 [arXiv: 1611.03874].

[11] ICECuBE collaboration, Searches for small-scale anisotropies from neutrino point sources with three years of Ice Cube data, Astropart. Phys. 66 (2015) 39 [arXiv: 1408.0634]. 
[12] ICECuBE collaboration, All-sky Search for Time-integrated Neutrino Emission from Astrophysical Sources with 7 yr of IceCube Data, Astrophys. J. 835 (2017) 151 [arXiv: 1609. 04981].

[13] ICECuBE collaboration, Neutrino emission from the direction of the blazar TXS 0506+056 prior to the IceCube-170922A alert, Science 361 (2018) 147 [arXiv: 1807. 08794].

[14] ICECube collaboration, The IceCube Realtime Alert System, Astropart. Phys. 92 (2017) 30 [arXiv: 1612.06028].

[15] D. Chirkin and W. Rhode, Muon Monte Carlo: A High-precision tool for muon propagation through matter, hep-ph/0407075.

[16] AMANDA collaboration, Muon track reconstruction and data selection techniques in AMANDA, Nucl. Instrum. Meth. A 524 (2004) 169 [astro-ph/0407044].

[17] T. Neunhoffer, Estimating the angular resolution of tracks in neutrino telescopes based on a likelihood analysis, Astropart. Phys. 25 (2006) 220 [astro-ph/0403367].

[18] L. Radel and C. Wiebusch, Calculation of the Cherenkov light yield from low energetic secondary particles accompanying high-energy muons in ice and water with Geant 4 simulations, Astropart. Phys. 38 (2012) 53 [arXiv: 1206.5530].

[19] V.J. Stenger, Track fitting for DUMAND-II Octagon Array, DUMAND External Report HDC-1-90 (1990) [https://www.phys.hawaii.edu/dumand/external_reports.html].

[20] M.G. Aartsen et al., Improvement in Fast Particle Track Reconstruction with Robust Statistics, Nucl. Instrum. Meth. A 736 (2014) 143 [arXiv: 1308. 5501].

[21] D. Pandel, Bestimmung von Wasser- und Detektorparametern und Rekonstruktion von Myonen bis $100 \mathrm{TeV}$ mit dem Baikal-Neutrinoteleskop NT-72, MSc Thesis, Humboldt-Universität, Berlin, Germany (1996).

[22] AMANDA collaboration, Muon track reconstruction and data selection techniques in AMANDA, Nucl. Instrum. Meth. A 524 (2004) 169 [astro-ph/0407044].

[23] N. van Eijndhoven, O. Fadiran and G. Japaridze, Implementation of a Gauss convoluted Pandel PDF for track reconstruction in Neutrino Telescopes, Astropart. Phys. 28 (2007) 456 [arXiv: 0704 . 1706].

[24] J. Lundberg, P. Miocinovic, T. Burgess, J. Adams, S. Hundertmark, P. Desiati et al., Light tracking for glaciers and oceans: Scattering and absorption in heterogeneous media with Photonics, Nucl. Instrum. Meth. A 581 (2007) 619 [astro-ph/0702108].

[25] N. Whitehorn, J. van Santen and S. Lafebre, Penalized Splines for Smooth Representation of High-dimensional Monte Carlo Datasets, Comput. Phys. Commun. 184 (2013) 2214 [arXiv: 1301.2184].

[26] K. Schatto, Stacked searches for high-energy neutrinos from blazars with IceCube, Ph.D. thesis, Mainz University (2014) [http://doi.org/10.25358/openscience-2897].

[27] F. James and M. Roos, Minuit: A System for Function Minimization and Analysis of the Parameter Errors and Correlations, Comput. Phys. Commun. 10 (1975) 343.

[28] P. Getreuer, A survey of gaussian convolution algorithms, Image Processing On Line 3 (2013) 286.

[29] S.S. Wilks, The large-sample distribution of the likelihood ratio for testing composite hypotheses, Ann. Math. Statist. 9 (1938) 60

[30] G. Cowan, Statistical Data Analysis, Oxford Science Publications, Clarendon Press (1998).

[31] D. Foreman-Mackey, D.W. Hogg, D. Lang and J. Goodman, emcee: The MCMC Hammer, Publ. Astron. Soc. Pac. 125 (2013) 306 [arXiv: 1202 . 3665]. 